Argonne

ANL/CFCT-19/6

\title{
Molten Salt Thermophysical Properties Database Development: 2019 Update
}

Chemical and Fuel Cycle Technologies Division 


\title{
About Argonne National Laboratory
}

Argonne is a U.S. Department of Energy laboratory managed by UChicago Argonne, LLC under contract DE-AC02-06CH11357. The Laboratory's main facility is outside Chicago, at 9700 South Cass Avenue, Argonne, Illinois 60439. For information about Argonne and its pioneering science and technology programs, see www.anl.gov.

\section{DOCUMENT AVAILABILITY}

Online Access: U.S. Department of Energy (DOE) reports produced after 1991 and a growing number of pre-1991 documents are available free at OSTI.GOV (http://www.osti.gov/), a service of the US Dept. of Energy's Office of Scientific and Technical Information.

\author{
Reports not in digital format may be purchased by the public from the \\ National Technical Information Service (NTIS): \\ U.S. Department of Commerce \\ National Technical Information Service \\ 5301 Shawnee Rd \\ Alexandria, VA 22312 \\ www.ntis.gov \\ Phone: (800) 553-NTIS (6847) or (703) 605-6000 \\ Fax: (703) 605-6900 \\ Email: orders@ntis.gov
}

Reports not in digital format are available to DOE and DOE contractors from the Office of Scientific and Technical Information (OSTI):

U.S. Department of Energy

Office of Scientific and Technical Information

P.O. Box 62

Oak Ridge, TN 37831-0062

www.osti.gov

Phone: (865) 576-8401

Fax: (865) 576-5728

Email: reports@osti.gov

\section{Disclaimer}

This report was prepared as an account of work sponsored by an agency of the United States Government. Neither the United States Government nor any agency thereof, nor UChicago Argonne, LLC, nor any of their employees or officers, makes any warranty, express or implied, or assumes any legal liability or responsibility for the accuracy, completeness, or usefulness of any information, apparatus, product, or process disclosed, or represents that its use would not infringe privately owned rights. Reference herein to any specific commercial product, process, or service by trade name, trademark, manufacturer, or otherwise, does not necessarily constitute or imply its endorsement, recommendation, or favoring by the United States Government or any agency thereof. The views and opinions of document authors expressed herein do not necessarily state or reflect those of the United States Government or any agency thereof, Argonne National Laboratory, or UChicago Argonne, LLC. 


\section{Molten Salt Thermophysical Properties Database Development: 2019 update}

prepared by James Jerden

Chemical and Fuel Cycle Technologies Division, Argonne National Laboratory

prepared for the U.S. DOE Advanced Reactor Technology Program's Molten Salt Reactor campaign, Salt Chemistry Work Package: AT-19AN04060104, Milestone: M4AT-19AN040601045.

$9 / 9 / 2019$ 


\section{Summary}

This report provides an update on the molten salt reactor thermophysical properties database that is being developed as part of the U.S. Department of Energy Advanced Reactor Technology Program Molten Salt Reactor (MSR) campaign. A summary of recent MSR-relevant literature and a discussion of data gaps are provided. The structure of the new MSR thermophysical properties database is outlined and three datasets are reviewed in detail to provide examples of the data and metadata that will be included in the database. Despite the availability of some high quality MSR-relevant data-sets, there remains a need for a thermophysical properties experimental program to generate data focused on addressing the design and licensing needs of current MSR concept developers.

This report represents the work product milestone M4AT-19AN040601045 for work package Salt Chemistry work package AT-19AN04060104 of the Advanced Reactor Technology Program's MSR campaign. 


\section{Table of Contents}

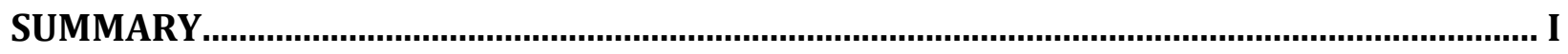

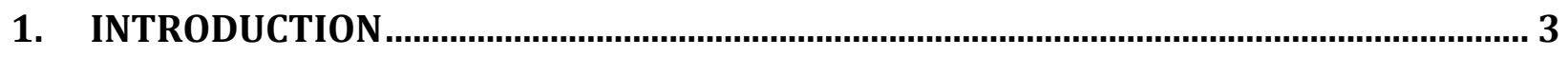

2. SUMMARY OF INITIAL LITERATURE REVIEW ............................................................ 4

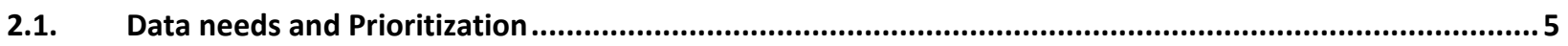

3. MSR THERMOPHYSICAL PROPERTIES DATABASE...................................................10

3.1. MSR Thermophysical Properties Database Inputs: Example Data Sets .................................................11

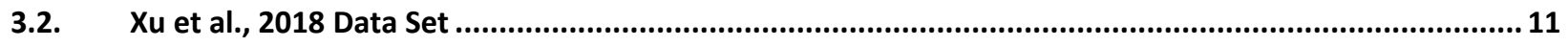

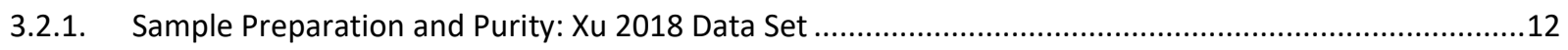

3.2.2. Heat Capacity Measurements: $\mathrm{Xu} 2018 \mathrm{MgCl}_{2}-\mathrm{KCl}$ Data Set ............................................................12

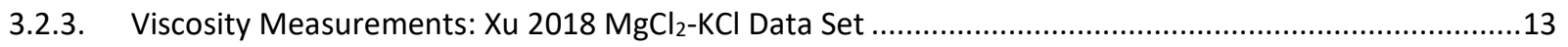

3.2.4. Density Measurements: $\mathrm{Xu} 2018 \mathrm{MgCl}_{2}-\mathrm{KCl}$ Data Set ...............................................................14

3.2.5. Thermal Conductivity Measurements: Xu $2018 \mathrm{MgCl}_{2}-\mathrm{KCl}$ Data Set..................................................15

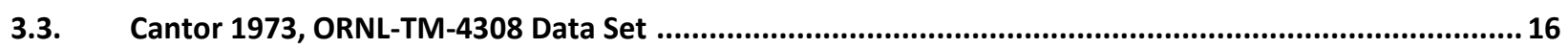

3.3.1. Sample Preparation and Purity: Cantor 1973, ORNL-TM-4308 Data Set...........................................16

3.3.2. Density Measurements: Cantor 1973, ORNL-TM-4308 Data Set......................................................17

3.3.3. Viscosity Measurements: Cantor 1973, ORNL-TM-4308 Data Set...................................................18

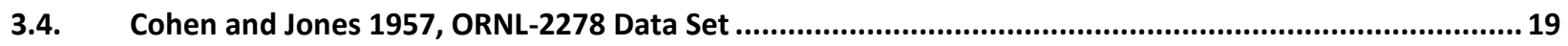

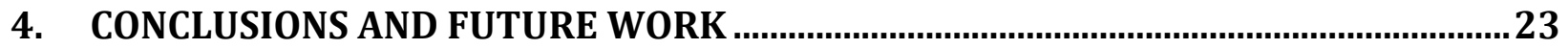

REFERENCES 


\section{Introduction}

The U.S. Department of Energy Advanced Reactor Technology Program's Molten Salt Reactor (MSR) campaign is developing state-of-the-art thermochemical and thermophysical properties databases to aid in the design and licensing of new MSRs. This report provides a progress update on the development of the molten salt thermophysical properties database.

The ultimate goal of the thermophysical properties database activity is to develop a state-of-the-art information tool that provides the fuel/coolant salt properties needed to model MSR performance as a function of temperature, pressure and salt composition. The database will be integrated into the systemlevel modeling and simulation tools being developed as part of the MSR campaign to assess and optimize the performance of particular MSR concepts. The specific content of the database will include experimentally measured thermophysical properties (e.g., density, viscosity, heat capacity and thermal conductivity) as well as predictive functions for the interpolation and extrapolation of those properties. The envisioned relationship between the thermophysical database and other components of the MSR modeling and simulation framework is shown Figure 1.

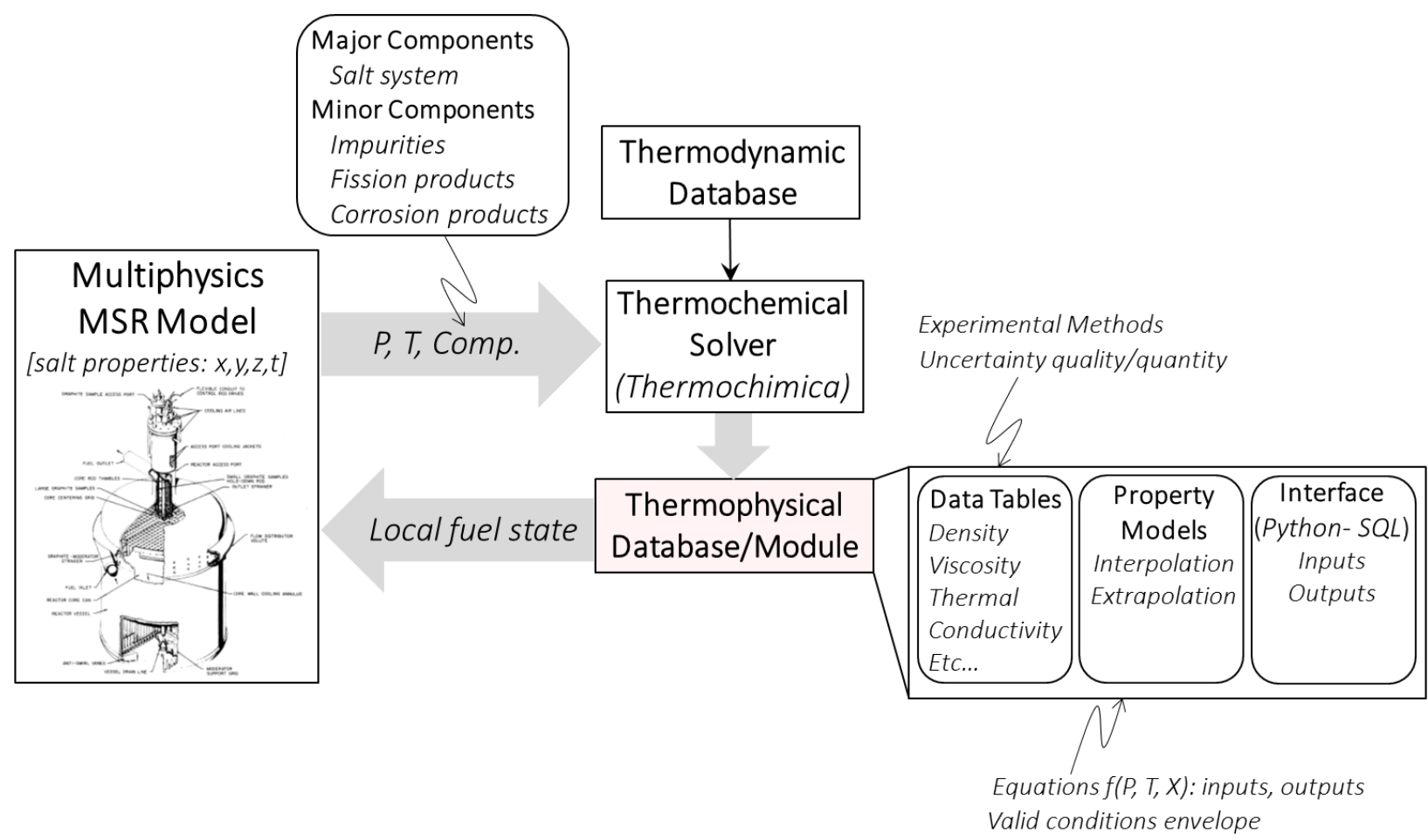

Figure 1. Diagram showing the conceptual relationships between the thermophysical properties database and the thermochemical and multiphysics MSR models.

To date, the thermophysical properties database development activity has involved critical reviews of relevant literature to provide the initial database content framework. The results of this literature review provide the initial datasets for populating the database and identify specific data gaps that provide objectives 
for future R\&D activities. Database development will require close coordination and discussions between molten salt reactor developers, systems engineers, modeling and simulation experts, chemists and chemical engineers to ensure the database supports project needs.

\section{Summary of Initial Literature Review}

This section presents an overview of the state of knowledge regarding the essential thermophysical properties of MSR-relevant salt systems. The goal of this section is to provide the technical basis for identifying and prioritizing future research and development activities that are needed to compile a thermophysical properties database that can be directly integrated into reactor performance codes.

There are three general applications of molten salts in these advanced nuclear reactor systems: fuel, primary coolant, and heat exchange medium. The fuel and coolant salts consist of binary, ternary or quaternary salt mixtures that contain the fissile actinides that fission in the reactor core and are circulated within the primary loop to the primary heat exchangers during reactor operation (e.g., Samuel, 2009). Heat from the primary heat exchangers may then be transferred to a secondary coolant salt that does not contain fissile or fertile elements. In salt-cooled solid fueled reactors (e.g., liquid salt cooled reactors), the molten salt is restricted to circulation within the primary cooling loop to transport heat from the core to intermediate loops that may also use liquid salts to transport heat to either a Brayton power cycle for electricity production or to a hydrogen production facility (Samuel, 2009, Forsberg et al., 2008).

Table 1 lists examples of molten salt compositions proposed for applications in Generation IV nuclear reactor technologies. 
Table 1. Examples of applications of molten salts in proposed and tested nuclear reactor concepts (adapted from Samuel, 2009).

\begin{tabular}{|c|c|c|c|c|}
\hline Reactor Type & $\begin{array}{l}\text { Neutron } \\
\text { Spectrum }\end{array}$ & $\begin{array}{l}\text { Molten Salt } \\
\text { Application }\end{array}$ & Reference Salt Systems & Selected References \\
\hline \multirow{5}{*}{$\begin{array}{l}\text { Molten Salt } \\
\text { Breeder Reactor }\end{array}$} & Thermal & Fuel & ${ }^{7} \mathrm{LiF}-\mathrm{BeF}_{2}-\mathrm{AnF}_{4}$ & $\begin{array}{l}\text { [Grimes, 1967] } \\
{[\text { Furukawa, al. 2007] }} \\
\text { [Auger, et al. 2008] }\end{array}$ \\
\hline & \multirow{4}{*}{ Fast } & $\begin{array}{l}\text { Secondary } \\
\text { coolant }\end{array}$ & $\mathrm{NaF}_{-} \mathrm{NaBF}_{4}$ & $\begin{array}{l}\text { [Grimes, 1967] } \\
\text { [Furukawa, et al. 2007] } \\
\text { [Auger, et al. 2008] }\end{array}$ \\
\hline & & \multirow{3}{*}{ Fuel } & ${ }^{7} \mathrm{LiF}-\mathrm{AnF} 4$ & $\begin{array}{l}\text { [Grimes, 1967] } \\
{[\text { Furukawa, al. 2007] }} \\
\text { [Auger, et al. 2008] }\end{array}$ \\
\hline & & & $\mathrm{NaCl}-\mathrm{MgCl}_{2}-\mathrm{UCl}_{3}-\mathrm{PuCl}_{3}$ & [Beneš, Konings, 2009] \\
\hline & & & $\mathrm{LiF}-\mathrm{NaF}-\mathrm{BeF}_{2}-\mathrm{AnF}_{3}$ & [Ignatiev, et al. 2003]] \\
\hline $\begin{array}{l}\text { Advanced High } \\
\text { Temperature Reactor }\end{array}$ & Thermal & $\begin{array}{l}\text { Primary } \\
\text { coolant }\end{array}$ & ${ }^{7} \mathrm{LiF}_{-} \mathrm{BeF}_{2}$ & $\begin{array}{l}\text { [Ingersoll, et al. 2004] } \\
\text { [Williams, et al.2006] }\end{array}$ \\
\hline $\begin{array}{l}\text { Very High } \\
\text { Temperature Reactor }\end{array}$ & Thermal & $\begin{array}{l}\text { Heat transfer } \\
\text { coolant }\end{array}$ & LiF-NaF-KF & [Williams, 2006] \\
\hline \multirow{2}{*}{$\begin{array}{l}\text { Liquid Salt Cooled } \\
\text { Fast Reactor }\end{array}$} & \multirow{2}{*}{ Fast } & $\begin{array}{l}\text { Primary } \\
\text { coolant }\end{array}$ & LiCl-NaCl-MgCl 2 & [Forsberg et al. 2008] \\
\hline & & $\begin{array}{l}\text { Intermediate } \\
\text { coolant }\end{array}$ & $\mathrm{NaNO}_{3}-\mathrm{KNO}_{3}$ & [Latgé, 2008] \\
\hline
\end{tabular}

The specific salt systems and compositions that are best suited for particular applications will be based on their thermophysical and chemical properties. Several thermodynamic and physical properties databases for molten salts have been assembled and several review papers and reports summarize the substantial amount of literature in this field (e.g., Williams, 2006, Williams et al., 2006, Beneš, and Konings, 2009, Samuel, 2009, Serrano-López et al., 2013). However, there remains a need for a functional database and computational tool that can be used to select, optimize and predict the properties of specific molten salt mixtures being considered for current nuclear reactor applications.

In general, the MSR Campaign will focus on binary, ternary and quaternary salt mixtures because the melting points of individual salts are too high for coolant/fuel applications. A number of appropriate chloride and fluoride salt systems with eutectic behavior have been identified for use as coolants and fuel in different types of nuclear reactors (see Table 1 and references cited therein). However, there remains a need for a functional database that can support salt mixture properties modeling over the full range of relevant P-T-X (pressure, temperature, composition) conditions to optimize and assess the performance of MSR designs.

\subsection{Data needs and Prioritization}

A coolant or fuel-coolant salt must efficiently extract heat from the core at relatively high temperatures and efficiently transfer that heat to heat exchangers and energy conversion systems (e.g., steam generator- 
turbine system) without introducing unmanageable safety or waste issues. Examples of some of the key requirements include:

- Neutron attenuation: the salt should not significantly attenuate neutrons.

- Activation: the salt should not produce significant quantities of radioactive activation products that could complicate regular operation tasks (low neutron absorption cross sections) or substantially increase the source term.

- Corrosion: the salt should not be aggressively corrosive to the materials of construction used for core or loop components.

- Actinide solubility: the solubility of uranium, thorium and plutonium in a candidate fuel-coolant salt should be understood and predictable over relevant temperature ranges.

- Salt freezing: the salt must have a melting point that is at least $50^{\circ} \mathrm{C}$ lower than the lowest temperature that will occur within the coolant loop circuit during operation.

- Salt decomposition or boiling: the salt must be thermally stable at temperatures well above those anticipated during normal and off-normal operation (i.e., under credible accident conditions).

- Pumpability: the salt should have a reasonably low viscosity at operating temperatures to allow predictable flow dynamics.

- Vaporization: the salt should have a relatively low vapor pressure to avoid pressurization, aerosol generation and coolant loss issues at operating temperatures.

- Cost and materials availability: salt mixture components should be relatively inexpensive and readily available to accommodate scale up and commercialization.

- Opacity: the salt should ideally have low opacity to allow for with optical in-service inspection of core and coolant loop components.

- Toxicity: the coolant chemistry should not pose unnecessary toxicity hazard during handling for routine repair operations.

In addition to these general considerations, Sohal et al., 2013 identify the following specific qualities as essential criteria for assessing the applicability of molten salt systems for nuclear applications.

- Chemical stability of salt mixture at elevated temperatures $\left(500-800{ }^{\circ} \mathrm{C}\right)$.

- Radiolytic stability of salt mixture in high radiation fields (specific to primary coolant).

- Low freezing temperature, preferably lower than $500{ }^{\circ} \mathrm{C}$.

- Large heat capacity and thermal conductivity.

- Low vapor pressures: less than one atmosphere at operating temperatures.

- Compatible with reactor core/heat exchanger materials: alloys, graphite, and ceramics.

Fuel-containing salts have additional performance criteria because, in addition to carrying the fissile and fertile material, the salt may be required to act as a neutron moderator, provide negative thermal reactivity feedback, act as the primary heat transfer medium and provide decay heat removal by natural circulation (Holcomb et al., 2011). Therefore, in addition to the requirements listed above, fuel salts also have the following requirements:

- Very low concentrations of isotopes with high-parasitic neutron absorption cross sections within the relevant spectral region for a given reactor.

- Large coefficient of thermal expansion to provide strong negative reactivity feedback and efficient natural circulation.

- Ability to maintain high dissolved concentrations of fissile and fertile materials ( $\mathrm{U}, \mathrm{Pu}, \mathrm{Th})$ to support criticality and efficient breeding, if needed. 
The development of screening factors or operational criteria for salt systems will depend on the specific application within the reactor system. For example, previous studies have suggested that chloride salts are problematic for fast reactor applications due to their relatively high thermal-neutron absorption cross sections and undesirable neutron activation properties (e.g., ${ }^{36} \mathrm{Cl}$ ) (Williams, 2006, Williams et al., 2006, Samuel, 2009). However, recent work indicates that chloride salt systems such as $\mathrm{NaCl}-\mathrm{MgCl}_{2}$ containing fertile ${ }^{238} \mathrm{UCl}_{3}$ and fissile ${ }^{239} \mathrm{PuCl}_{3}$ show considerable promise as a fuel-coolant salt for fast breeder molten salt reactors (e.g., Mourogov and Bokov, 2006, Beneš, Konings, 2009).

A wealth of knowledge on the thermophysical properties of molten salts was gained at Oak Ridge National Laboratory in the 1950s - 1970s during the Aircraft Reactor Experiment (ARE), Molten Salt Reactor Experiment (MSRE) and the Molten Salt Breeder Reactor (MSBR) projects (Williams, 2006, Williams et al., 2006, Samuel, 2009). More recently, work in the Advanced High-Temperature Reactor (AHTR), Next Generation Nuclear Plant (NGNP) and Nuclear Hydrogen Initiative (NHI) programs has provided important information about molten salt property criteria for nuclear applications (for example, Ingersoll 2004, Williams, 2006, Williams et al., 2006). Other key information relevant to molten salt applications as coolants and fuels in MSRs has been compiled in the European Molten Salt Technology (MOST) database (Renault and Delpech, M., 2005) and work in Russia described by Ignatiev et al., 2002, Ignatiev et al., 2003, Khokhlov et al., 2009. Furthermore, general salt properties information was made available online through the Molten Salt Database (MSDB) project (http://allen.neep.wisc.edu/shell/), which was a collaborative effort involving the University of Wisconsin and Shell Oil Company.

In addition to these nuclear application-focused databases, Janz and others compiled and maintained an extensive database of thermophysical and thermochemical property values for molten salts relevant for energy storage (Janz et al., 1968, - Janz, 1988). The Janz database includes a number of salt compositions that may be applicable as MSR coolants, but actinide-bearing salt compositions are not represented.

Tables 2 and 3 give melting point, boiling point, vapor pressures, densities, heat capacities, viscosities and thermal conductivities for some of the major candidate molten salt systems. The cells identified as "Data Need" indicate information gaps for these salt compositions. It is likely that new experimental work will be needed to fill these information gaps to further consider use of those mixtures. As pointed out by Williams et al., 2006, melting points, vapor pressures and viscosities can be measured accurately, but it is difficult to predict these property values accurately by using current parameter databases. There is a particular need for high temperature heat capacity, viscosity and thermal conductivity data for many relevant salt systems to establish accurate equations for temperature dependencies to predict values throughout the range of operating temperatures.

Table 4 lists a number of possible MSR fuel salts that have been the subject of recent thermodynamic studies by the European Commission's Institute for Transuranium Elements (ITU). For example, Capelli, 2013 reports a number of advances in measuring thermophysical and thermochemical properties of MSRrelevant salt mixtures at elevated temperatures. Table 4 summarizes the results for salt systems that are discussed in some detail by Capelli, 2013. 
Table 2. Thermophysical properties extracted from literature for MSR-relevant salts (adapted from Samuel, 2009).

\begin{tabular}{|c|c|c|c|c|c|}
\hline \multicolumn{2}{|l|}{ Salt Mixture } & \multirow{2}{*}{$\begin{array}{c}\text { Melting } \\
\text { Point or } \\
\text { *Liquidus T } \\
\left({ }^{\circ} \mathrm{C}\right) \\
\end{array}$} & \multirow{2}{*}{$\begin{array}{c}\text { Boiling } \\
\text { Point } \\
\left({ }^{\circ} \mathrm{C}\right)\end{array}$} & \multirow{2}{*}{$\begin{array}{c}\text { Vapor Pressure } \\
(\mathrm{mm} \mathrm{Hg}) \text { at } 900^{\circ} \mathrm{C} \\
\text { or Function }\end{array}$} & \multirow[t]{2}{*}{ Density $\left(\mathrm{g} / \mathrm{cm}^{3}\right)$} \\
\hline Type & Composition (mol\%) & & & & \\
\hline \multirow[t]{4}{*}{$\begin{array}{l}\text { Alkali } \\
\text { Fluorides }\end{array}$} & $\begin{array}{l}\mathrm{LiF}-\mathrm{KF} \\
(50-50)\end{array}$ & 492 & $\begin{array}{l}\text { Data } \\
\text { Need }\end{array}$ & Data Need & $2.460-0.00068 \cdot \mathrm{T}\left(847-1067^{\circ} \mathrm{C}\right)$ \\
\hline & $\begin{array}{l}\text { LiF-RbF } \\
(44-56)\end{array}$ & 470 & $\begin{array}{l}\text { Data } \\
\text { Need }\end{array}$ & Data Need & $3.300-0.00096 \bullet \mathrm{T}\left(500-750^{\circ} \mathrm{C}\right)$ \\
\hline & $\begin{array}{l}\text { LiF-NaF-KF } \\
(46.5-11.5-42)\end{array}$ & 454 & 1570 & -0.7 & $2.530-0.00073 \cdot \mathrm{T}\left(940-1170^{\circ} \mathrm{C}\right)$ \\
\hline & $\begin{array}{l}\text { LiF-NaF-RbF } \\
(42-6-52)\end{array}$ & 435 & $\begin{array}{l}\text { Data } \\
\text { Need }\end{array}$ & -0.8 & $3.261-0.000811 \cdot \mathrm{T}\left(500-700^{\circ} \mathrm{C}\right)$ \\
\hline \multirow[t]{4}{*}{$\mathrm{ZrF}_{4}$ Salts } & $\begin{array}{l}\mathrm{LiF}-\mathrm{ZrF} 4 \\
(51-49)\end{array}$ & 509 & $\begin{array}{l}\text { Data } \\
\text { Need }\end{array}$ & 77 & $3.739-0.000924 \cdot \mathrm{T}$ \\
\hline & $\begin{array}{l}\mathrm{NaF}-\mathrm{ZrF} 4 \\
(59.5-40.5)\end{array}$ & 500 & 1350 & 5 & $3.650-0.00088 \cdot \mathrm{T}\left(550-700^{\circ} \mathrm{C}\right)$ \\
\hline & $\begin{array}{l}\mathrm{RbF}-\mathrm{ZrF}_{4} \\
(58-42)\end{array}$ & 410 & 1450 & 1.3 & $3.923-0.00100 \bullet \mathrm{T}$ \\
\hline & $\begin{array}{l}\text { LiF-NaF-ZrF } 4 \\
(26-37-37)\end{array}$ & 436 & $\begin{array}{l}\text { Data } \\
\text { Need }\end{array}$ & 5 & $3.533-0.00087 \cdot \mathrm{T}\left(500-650^{\circ} \mathrm{C}\right)$ \\
\hline \multirow[t]{3}{*}{$\mathrm{BeF}_{2}$ Salts } & $\begin{array}{l}\text { LiF-BeF2 } \\
(67-33)\end{array}$ & 460 & 1400 & Data Need & $2.280-0.000488 \cdot \mathrm{T}\left(500-650^{\circ} \mathrm{C}\right)$ \\
\hline & $\begin{array}{l}\mathrm{NaF}-\mathrm{BeF}_{2} \\
(57-43)\end{array}$ & 340 & 1400 & 1.4 & $2.270-0.00037 \cdot \mathrm{T}\left(450-550^{\circ} \mathrm{C}\right)$ \\
\hline & $\begin{array}{l}\text { LiF-NaF-BeF } 2 \\
(31-31-38)\end{array}$ & 315 & $\begin{array}{l}\text { Data } \\
\text { Need }\end{array}$ & 1.7 & $2.313-0.000450 \cdot \mathrm{T}\left(450-550^{\circ} \mathrm{C}\right)$ \\
\hline \multirow[t]{4}{*}{ Chlorides } & $\begin{array}{l}\mathrm{LiCl}-\mathrm{KCl} \\
(59.5-40.5)\end{array}$ & 355 & 1400 & 5.8 & $1.8772-0.00087 \cdot \mathrm{T}$ \\
\hline & $\begin{array}{l}\mathrm{LiCl}-\mathrm{RbCl} \\
(58-42)\end{array}$ & 313 & $\begin{array}{l}\text { Data } \\
\text { Need }\end{array}$ & Data Need & $2.7416-0.000689 \cdot \mathrm{T}$ \\
\hline & $\begin{array}{l}\mathrm{NaCl}-\mathrm{MgCl}_{2} \\
(68-32)\end{array}$ & 445 & 1465 & $<2.5$ & $2.2971-0.000507 \cdot \mathrm{T}$ \\
\hline & $\begin{array}{l}\mathrm{KCl}-\mathrm{MgCl}_{2} \\
(68-32)\end{array}$ & 426 & $>1418$ & $<2$ & $2.2546-0.000474 \cdot \mathrm{T}$ \\
\hline \multirow[t]{3}{*}{$\begin{array}{l}\text { Fluoroborate } \\
\text { Salts }\end{array}$} & $\begin{array}{l}\mathrm{NaF}-\mathrm{NaBF}_{4} \\
(8-92)\end{array}$ & 385 & 694 & 9500 & $2.2521-0.000711 \cdot \mathrm{T}$ \\
\hline & $\begin{array}{l}\text { KF-KBF } 4 \\
(25-75)\end{array}$ & 460 & 1070 & 100 & $2.258-0.0008026 \bullet \mathrm{T}$ \\
\hline & $\begin{array}{l}\text { RbF-RbBF } 4 \\
(31-69)\end{array}$ & 442 & 1070 & $<100$ & $2.946-0.001047 \bullet \mathrm{T}$ \\
\hline \multirow{4}{*}{$\begin{array}{l}\text { MSR } \\
\text { Breeder Fuel } \\
\text { Salts }\end{array}$} & $\begin{array}{l}\text { LiF-BeF2-ThF4-UF } 4 \\
(73-16-10.7-0.3)\end{array}$ & 500 & $\begin{array}{l}\text { Data } \\
\text { Need }\end{array}$ & $\begin{array}{l}8.0-10000 / \mathrm{T}(\mathrm{K}) \\
\left(500-700^{\circ} \mathrm{C}\right)\end{array}$ & $3.628-0.00066 \cdot \mathrm{T}\left({ }^{\circ} \mathrm{C}\right)$ \\
\hline & $\begin{array}{l}\text { LiF-BeF2-ThF4-UF4 } \\
(72-21-6.7-0.3)\end{array}$ & $500^{*}$ & $\begin{array}{l}\text { Data } \\
\text { Need }\end{array}$ & $\begin{array}{c}8.0-10000 / \mathrm{T}(\mathrm{K}) \\
\left(500-700^{\circ} \mathrm{C}\right)\end{array}$ & $3.153-0.00058 \cdot \mathrm{T}\left({ }^{\circ} \mathrm{C}\right)$ \\
\hline & $\begin{array}{l}\text { LiF-BeF2-ThF4-UF4 } \\
(68-20-11-7-0.3)\end{array}$ & $480^{*}$ & $\begin{array}{l}\text { Data } \\
\text { Need }\end{array}$ & $\begin{array}{l}8.0-10000 / \mathrm{T}(\mathrm{K}) \\
\left(500-700^{\circ} \mathrm{C}\right)\end{array}$ & $3.644-0.00063 \cdot \mathrm{T}\left({ }^{\circ} \mathrm{C}\right)$ \\
\hline & $\begin{array}{l}\text { LiF-BeF2-ThF4-UF } 4 \\
(63-25-11.7-0.3)\end{array}$ & $500 *$ & $\begin{array}{l}\text { Data } \\
\text { Need }\end{array}$ & $\begin{array}{l}8.0-10000 / \mathrm{T}(\mathrm{K}) \\
\left(500-700^{\circ} \mathrm{C}\right)\end{array}$ & $3.628-0.00066 \cdot \mathrm{T}\left({ }^{\circ} \mathrm{C}\right)$ \\
\hline
\end{tabular}


Table 3. thermophysical properties extracted from literature for MSR-relevant salts (adapted from Samuel, 2009).

\begin{tabular}{|c|c|c|c|c|c|c|c|}
\hline \multicolumn{2}{|c|}{ Salt Mixture } & \multicolumn{2}{|c|}{$\begin{array}{c}\text { Heat Capacity } \\
\left(\mathrm{cal} / \mathrm{g}^{\circ} \mathrm{C}\right)\end{array}$} & \multicolumn{2}{|c|}{ Viscosity (cP) } & \multicolumn{2}{|c|}{$\begin{array}{c}\text { Thermal Conductivity } \\
(\mathrm{W} / \mathrm{m} \mathrm{K})\end{array}$} \\
\hline Type & Composition $(\mathrm{mol} \%)$ & $\begin{array}{c}\text { Measured } \\
\left(700^{\circ} \mathrm{C}\right)\end{array}$ & $\begin{array}{c}\text { Predicted } \\
\left(700^{\circ} \mathrm{C}\right)\end{array}$ & $\begin{array}{l}\text { Measured } \\
\left(700^{\circ} \mathrm{C}\right)\end{array}$ & T Function & $\begin{array}{l}\text { Predicted } \\
\left(700^{\circ} \mathrm{C}\right)\end{array}$ & Measured \\
\hline \multirow[t]{4}{*}{$\begin{array}{l}\text { Alkali } \\
\text { Fluorides }\end{array}$} & $\begin{array}{l}\mathrm{LiF}-\mathrm{KF} \\
(50-50)\end{array}$ & 0.44 & 0.381 & $\begin{array}{l}\text { Data } \\
\text { Need }\end{array}$ & $\begin{array}{l}\text { Data } \\
\text { Need }\end{array}$ & 0.91 & $\begin{array}{l}\text { Data } \\
\text { Need }\end{array}$ \\
\hline & $\begin{array}{l}\mathrm{LiF}-\mathrm{RbF} \\
(44-56)\end{array}$ & 0.284 & 0.226 & $\begin{array}{c}\text { See } \\
\text { Function }\end{array}$ & $\begin{array}{c}0.0212 \exp (4678 / \mathrm{T}) \\
(873-1073 \mathrm{~K})\end{array}$ & $T B A$ & $\begin{array}{l}\text { Data } \\
\text { Need }\end{array}$ \\
\hline & $\begin{array}{l}\mathrm{LiF}-\mathrm{NaF}-\mathrm{KF} \\
(46.5-11.5-42)\end{array}$ & 0.45 & 0.387 & 2.9 & $\begin{array}{c}0.04 \exp (4170 / \mathrm{T}) \\
(873-1073 \mathrm{~K})\end{array}$ & 0.92 & $\begin{array}{c}0.6 \\
\left(500^{\circ} \mathrm{C}\right)\end{array}$ \\
\hline & $\begin{array}{l}\text { LiF-NaF-RbF } \\
(42-6-52)\end{array}$ & $\begin{array}{l}\text { Data } \\
\text { Need }\end{array}$ & 0.236 & 2.6 & $\begin{array}{l}\text { Data } \\
\text { Need }\end{array}$ & 0.62 & $\begin{array}{l}\text { Data } \\
\text { Need }\end{array}$ \\
\hline \multirow[t]{4}{*}{$\mathrm{ZrF}_{4}$ Salts } & $\begin{array}{l}\mathrm{LiF}-\mathrm{ZrF}{ }_{4} \\
(51-49)\end{array}$ & $\begin{array}{l}\text { Data } \\
\text { Need }\end{array}$ & 0.292 & $>5.1$ & $\begin{array}{l}\text { Data } \\
\text { Need }\end{array}$ & 0.48 & $\begin{array}{l}\text { Data } \\
\text { Need }\end{array}$ \\
\hline & $\begin{array}{l}\mathrm{NaF-ZrF} 4 \\
(59.5-40.5)\end{array}$ & 0.28 & 0.275 & 5.1 & $\begin{array}{c}0.0767 \exp (3977 / \mathrm{T}) \\
(873-1073 \mathrm{~K})\end{array}$ & 0.49 & $\begin{array}{l}\text { Data } \\
\text { Need }\end{array}$ \\
\hline & $\begin{array}{l}\mathrm{RbF}-\mathrm{ZrF}_{4} \\
(58-42)\end{array}$ & $\begin{array}{l}\text { Data } \\
\text { Need }\end{array}$ & 0.2 & 5.1 & $\begin{array}{l}\text { Data } \\
\text { Need }\end{array}$ & 0.39 & $\begin{array}{l}\text { Data } \\
\text { Need }\end{array}$ \\
\hline & $\begin{array}{l}\text { LiF-NaF-ZrF } 4 \\
(26-37-37)\end{array}$ & $\begin{array}{l}\text { Data } \\
\text { Need }\end{array}$ & 0.296 & 6.9 & $\begin{array}{l}\text { Data } \\
\text { Need }\end{array}$ & 0.53 & $\begin{array}{l}\text { Data } \\
\text { Need }\end{array}$ \\
\hline \multirow[t]{3}{*}{$\mathrm{BeF}_{2}$ Salts } & $\begin{array}{l}\mathrm{LiF}-\mathrm{BeF}_{2} \\
(67-33)\end{array}$ & 0.577 & 0.566 & 5.6 & $\begin{array}{c}0.0116 \exp (3755 / \mathrm{T}) \\
(873-1073 \mathrm{~K})\end{array}$ & 1.1 & $\begin{array}{c}1.0 \\
\left(600^{\circ} \mathrm{C}\right)\end{array}$ \\
\hline & $\begin{array}{l}\mathrm{NaF}-\mathrm{BeF}_{2} \\
(57-43)\end{array}$ & 0.52 & 0.44 & 7 & $\begin{array}{c}0.0346 \exp (5165 / \mathrm{T}) \\
(873-1073 \mathrm{~K})\end{array}$ & 0.87 & 1 \\
\hline & $\begin{array}{l}\mathrm{LiF}-\mathrm{NaF}-\mathrm{BeF}_{2} \\
(31-31-38)\end{array}$ & $\begin{array}{l}\text { Data } \\
\text { Need }\end{array}$ & 0.489 & 5 & $\begin{array}{l}\text { Data } \\
\text { Need }\end{array}$ & 0.97 & $T B A$ \\
\hline \multirow[t]{4}{*}{ Chlorides } & $\begin{array}{l}\mathrm{LiCl}-\mathrm{KCl} \\
(59.5-40.5)\end{array}$ & 0.287 & 0.289 & 1.15 & $\begin{array}{c}0.0861 \exp (2517 / \mathrm{T}) \\
(873-1173 \mathrm{~K})\end{array}$ & 0.42 & $\begin{array}{c}0.38 \\
\left(700^{\circ} \mathrm{C}\right)\end{array}$ \\
\hline & $\begin{array}{l}\mathrm{LiCl}-\mathrm{RbCl} \\
(58-42)\end{array}$ & 0.213 & 0.212 & 1.3 & $\begin{array}{l}\text { Data } \\
\text { Need }\end{array}$ & 0.36 & $\begin{array}{l}\text { Data } \\
\text { Need }\end{array}$ \\
\hline & $\begin{array}{l}\mathrm{NaCl}-\mathrm{MgCl}_{2} \\
(68-32)\end{array}$ & 0.258 & 0.262 & 1.36 & $\begin{array}{c}0.0286 \exp (1441 / \mathrm{T}) \\
(973-1173 \mathrm{~K})\end{array}$ & 0.5 & $\begin{array}{l}\text { Data } \\
\text { Need }\end{array}$ \\
\hline & $\begin{array}{l}\mathrm{KCl}-\mathrm{MgCl}_{2} \\
(68-32)\end{array}$ & 0.276 & 0.229 & 1.4 & $\begin{array}{c}0.0146 \exp (2230 / \mathrm{T}) \\
(900-1073 \mathrm{~K})\end{array}$ & Data Need & $\begin{array}{l}\text { Data } \\
\text { Need }\end{array}$ \\
\hline \multirow[t]{3}{*}{$\begin{array}{l}\text { Fluorobor } \\
\text { ate Salts }\end{array}$} & $\begin{array}{l}\mathrm{NaF}-\mathrm{NaBF}_{4} \\
(8-92)\end{array}$ & 0.36 & 0.435 & 0.9 & $\begin{array}{c}0.0877 \exp (2240 / \mathrm{T}) \\
(873-1173 \mathrm{~K}) \\
\end{array}$ & 0.4 & $\begin{array}{c}0.4 \\
\left(621^{\circ} \mathrm{C}\right) \\
\end{array}$ \\
\hline & $\begin{array}{l}\mathrm{KF}-\mathrm{KBF}_{4} \\
(25-75)\end{array}$ & 0.312 & 0.367 & 0.9 & $\begin{array}{c}0.0431 \exp (3060 / \mathrm{T}) \\
(873-1073 \mathrm{~K})\end{array}$ & 0.38 & $\begin{array}{l}\text { Data } \\
\text { Need }\end{array}$ \\
\hline & $\begin{array}{l}\mathrm{RbF}_{-} \mathrm{RbBF}_{4} \\
(31-69)\end{array}$ & 0.218 & 0.258 & 0.9 & $\begin{array}{l}\text { Data } \\
\text { Need }\end{array}$ & 0.28 & $\begin{array}{l}\text { Data } \\
\text { Need }\end{array}$ \\
\hline \multirow{4}{*}{$\begin{array}{l}\text { MSR } \\
\text { Breeder } \\
\text { Fuel Salts }\end{array}$} & 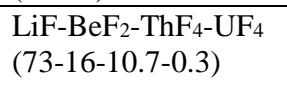 & 0.34 & $\begin{array}{l}\text { Data } \\
\text { Need }\end{array}$ & $\begin{array}{l}\text { Data } \\
\text { Need }\end{array}$ & $0.084 \exp (4340 / \mathrm{T})$ & 0.01 & $\begin{array}{l}\text { Data } \\
\text { Need }\end{array}$ \\
\hline & $\begin{array}{l}\mathrm{LiF}-\mathrm{BeF}_{2}-\mathrm{ThF}_{4}-\mathrm{UF}_{4} \\
(72-21-6.7-0.3)\end{array}$ & 0.39 & $\begin{array}{l}\text { Data } \\
\text { Need }\end{array}$ & $\begin{array}{l}\text { Data } \\
\text { Need }\end{array}$ & $0.072 \exp (4370 / \mathrm{T})$ & 0.01 & $\begin{array}{l}\text { Data } \\
\text { Need }\end{array}$ \\
\hline & $\begin{array}{l}\mathrm{LiF}-\mathrm{BeF}_{2}-\mathrm{ThF}_{4}-\mathrm{UF}_{4} \\
(68-20-11-7-0.3)\end{array}$ & 0.33 & $\begin{array}{l}\text { Data } \\
\text { Need }\end{array}$ & $\begin{array}{l}\text { Data } \\
\text { Need }\end{array}$ & $0.044 \exp (5030 / \mathrm{T})$ & 0.007 & $\begin{array}{l}\text { Data } \\
\text { Need }\end{array}$ \\
\hline & $\begin{array}{l}\mathrm{LiF} \mathrm{BeF}_{2}-\mathrm{ThF}_{4}-\mathrm{UF}_{4} \\
(63-25-11.7-0.3)\end{array}$ & 0.34 & $\begin{array}{l}\text { Data } \\
\text { Need }\end{array}$ & $\begin{array}{l}\text { Data } \\
\text { Need }\end{array}$ & $0.084 \exp (4340 / \mathrm{T})$ & 0.01 & $\begin{array}{l}\text { Data } \\
\text { Need }\end{array}$ \\
\hline
\end{tabular}


Table 4. fuel salt compositions studied by Capelli, 2013 (adapted from Capellie, 2013).

\begin{tabular}{|c|c|c|c|c|}
\hline Salt Mixture & Composition (mol\%) & $\begin{array}{c}\text { Liquidus T } \\
\quad\left({ }^{\circ} \mathrm{C}\right)\end{array}$ & Boiling $\mathbf{T}\left({ }^{\circ} \mathrm{C}\right)$ & $\begin{array}{c}\text { Vapor } P \text { at } 50 K \text { above } \\
\text { Melting } T(\mathrm{~Pa})\end{array}$ \\
\hline $\mathrm{LiF}_{-} \mathrm{ThF}_{4}$ & $76.2-23.8$ & 559 & 1754 & $8.56 \times 10^{-4}$ \\
\hline $\mathrm{Li}-\mathrm{ThF}_{4}-\mathrm{UF}_{4}-\mathrm{PuF}_{3}$ & $78.0-16.0-1.0-5.0$ & 594 & 1762 & $5.33 \times 10^{-3}$ \\
\hline $\mathrm{LiF}_{-} \mathrm{ThF}_{4}-\mathrm{UF}_{4}-\mathrm{PuF}_{3}$ & $75.3-20.6-1.0-3.1$ & 546 & 1759 & $7.26 \times 10^{-4}$ \\
\hline $\mathrm{LiF}_{-} \mathrm{ThF}_{4}-\mathrm{UF}_{4}$ & $76.2-21.3-2.55$ & 557 & 1749 & $1.08 \times 10^{-3}$ \\
\hline $\mathrm{LiF}-\mathrm{BeF}_{2}-\mathrm{ThF}_{4}-\mathrm{UF}_{4}$ & $75.7-1.8-19.95-2.55$ & 552 & 1718 & $4.67 \times 10^{-3}$ \\
\hline $\mathrm{LiF}_{-\mathrm{NaF}}-\mathrm{ThF}_{4}-\mathrm{UF}_{4}$ & $47.3-25.9-24.3-2.55$ & 497 & 1765 & $7.27 \times 10^{-5}$ \\
\hline $\mathrm{LiF}-\mathrm{NaF}-\mathrm{BeF}_{2}-\mathrm{ThF}_{4}-\mathrm{UF}_{4}$ & $41.8-31.1-4.6-19.95-2.55$ & 480 & 1706 & $4.08 \times 10^{-5}$ \\
\hline $\mathrm{LiF}-\mathrm{CaF}_{2}-\mathrm{ThF}_{4}$ & $66.9-4.3-28.8$ & 538 & 1744 & $4.12 \times 10^{-4}$ \\
\hline
\end{tabular}

Melting point and phase composition information can be readily extracted from accurate binary and ternary phase diagrams. Therefore, existing phase diagrams for relevant salt systems will be compiled, catalogued and indexed as part of the data compilation for this new MSR database development activity.

In addition to the properties identified in Tables 2 and 3, some databases (e.g., Janz 1988) include properties that are less commonly referenced but may be important for developing fundamental relationships between salt chemistry and thermophysical behavior, such as surface tension, electrical conductivity, bulk modulus, self-diffusivity of components and cryoscopic constants. These properties can be screened to determine if they provide essential functional needs within the thermophysical properties database to be integrated with MSR models.

\section{MSR Thermophysical Properties Database}

The initial MSR thermophysical properties database has been developed based on insights gained from the literature review summarized above. It consists of empirically derived functions of temperature for each thermophysical property to facilitate the use of the database within the reactor system model (as shown schematically in Figure 1 above). The columns of the current database are shown in Table 5. Each property column has a sub-columns for:

- Applicable temperature range,

- Percentage uncertainty,

- Literature reference or data report identifier for new experimental data.

Future versions of the database will allow different forms of the empirical expressions to be used, if need. 
Table 5. Exerpt showing main columns in the MSR thermophysical properties database. Each column contains sub-columns for the applicable temperature range, percentage uncertainty and references.

\begin{tabular}{|c|c|c|c|c|c|c|c|c|c|c|c|c|c|}
\hline \multirow[t]{2}{*}{ Salt } & \multirow[t]{2}{*}{$\begin{array}{c}\text { Composition } \\
(\text { mole } \%)\end{array}$} & \multirow{2}{*}{$\begin{array}{l}\text { Melting } \\
\text { Temp. } \\
(K)\end{array}$} & \multirow{2}{*}{$\begin{array}{l}\text { Boiling } \\
\text { Temp. } \\
(K)\end{array}$} & \multicolumn{2}{|c|}{$\begin{array}{l}\text { Density } \\
\left(\mathrm{g} / \mathrm{cm}^{3}\right) \\
A-B T\end{array}$} & \multicolumn{2}{|c|}{$\begin{array}{c}\text { Viscosity } \\
\text { (centipoise) } \\
A e^{B / T}\end{array}$} & \multicolumn{2}{|c|}{$\begin{array}{c}\text { Thermal } \\
\text { Conductivity } \\
\text { (W/m K) } \\
A+B T \\
\end{array}$} & \multicolumn{4}{|c|}{$\begin{array}{l}\text { Heat Capacity of Liquid } \\
(\mathrm{J} / \mathrm{K} \mathrm{mol}) \\
A+B T+C T^{-2}+D T^{2}\end{array}$} \\
\hline & & & & $A$ & $B$ & A & $B$ & $A$ & $B$ & $A$ & $B$ & $C$ & $D$ \\
\hline $\begin{array}{l}\text { LiF- } \\
\text { NaF- } \\
\mathrm{KF}\end{array}$ & $46.5-11.5-42.0$ & 727 & 1843 & 2.6 & $6.2 \times 10^{-4}$ & 0.025 & $4.5 \times 10^{3}$ & 0.36 & $5.6 \times 10^{-4}$ & 980 & 1.1 & & \\
\hline
\end{tabular}

To ensure robust quality assurance, all new data sets (from the literature or new experimental measurements) submitted for inclusion in the MSR thermophysical properties database will provide a standard set of data, error analyses and metadata (information on sample purity, preparation methods, instrument calibration, etc.) where possible so that the overall uncertainty in the data for a given salt system can be assessed and quantified. The following section describes three datasets that have been input into the database as examples.

\subsection{MSR Thermophysical Properties Database Inputs: Example Data Sets}

Three example data sets that have been added to the MSR thermophysical properties database are summarized to give examples of the information that should accompany data set entries. The key quantities and metadata include:

- Summary of salt sample preparation including sources and methods of mixing and fusion.

- Purification methods used.

- Estimation or quantification of purity.

- Summary description of thermophysical property measurement instrumentation and method.

- Summary of instrument calibration.

- Quantification of measurement errors.

- Quantification or estimate of overall measurement uncertainties.

Although data sets in the literature commonly do not quantify impurities in the salt, data produced from new experiments should include quantitative estimates or analyses of specific impurity elements in the samples.

The three data sets summarized below include the relatively recent work of $\mathrm{Xu}$ et al., 2018 on the $\mathrm{MgCl}_{2^{-}}$ $\mathrm{KCl}$ system, the work of Cantor, 1973 on the density and viscosity of zirconium, thorium and uranium bearing beryllium fluoride salts and the work of Cohen and Jones, 1957 on the viscosity of 36 binary, ternary and quaternary fluoride salts with various combinations of sodium, lithium, potassium, rubidium, beryllium, zirconium and uranium.

\subsection{Xu et al., 2018 Data Set}

The work of Xu et al., 2018 quantifies the heat capacity, viscosity, density, and thermal conductivity of $\mathrm{MgCl}_{2}-\mathrm{KCl}$ (32-68 mole \%). This salt is an important candidate material for use in MSR heat exchangers and as a high temperature heat transfer and storage medium for concentrated solar thermal power systems. 
The thermophysical properties for 32-68 mole $\% \mathrm{MgCl}_{2}-\mathrm{KCl}$ determined by $\mathrm{Xu}$ et al., 2018 are summarized in Table 6. The table provides the polynomial relationships derived from the data as well as the $95 \%$ confidence interval for the data correlation. The measurement instrumentation and methods are discussed below.

Table 6. Summary of empirical functions reported by Xu et al., for the thermophysical properties of 32-68 mole \% $\mathrm{MgCl}_{2}-\mathrm{KCl}$

\begin{tabular}{lll}
\hline Property & Values or Functions of $\mathbf{T}\left({ }^{\circ} \mathbf{C}\right)$ & $\mathbf{9 5 \%}$ Uncertainty \\
\hline Melting point $\left({ }^{\circ} \mathrm{C}\right)$ & 424.4 & 1.0 \\
Heat capacity $(\mathrm{J} / \mathrm{g} \mathrm{K})$ & $\mathrm{C}_{\mathrm{p}}=0.9896+1.046 \times 10^{-4}(\mathrm{~T}-430)$ & $\leq 4.91 \times 10^{-2}$ \\
Viscosity $($ centipoise $)$ & $\mu=14.965-0.0291 \mathrm{~T}+1.784 \times 10^{-5} \mathrm{~T}^{2}$ & $\leq 0.443$ \\
Density $\left(\mathrm{kg} / \mathrm{m}^{3}\right)$ & $\rho=1903.7-0.552 \mathrm{~T}$ & $\leq 61.9$ \\
Thermal conductivity $(\mathrm{W} / \mathrm{m} \mathrm{K})$ & $\mathrm{k}=0.5047-0.0001 \mathrm{~T}$ & $\leq 3.69 \times 10^{-2}$ \\
\hline
\end{tabular}

\subsection{1. $\quad$ Sample Preparation and Purity: Xu 2018 Data Set}

Anhydrous $\mathrm{MgCl}_{2}$ and $\mathrm{KCl}$ salts with $99 \%$ purity were procured from Alfa Aesar, ground by hand and dry mixed inside a humidity-controlled glove box to obtain a composition of 32-68 mole $\% \mathrm{MgCl}_{2}-\mathrm{KCl}_{\text {. The }}$ relative humidity in the glove box was maintained at values less than $3 \%$. The ground mixtures were heated to $500{ }^{\circ} \mathrm{C}$ and held at that temperature for 24 hours to ensure complete melting of the samples. The fused samples were then allowed to cool naturally, ground into powders and stored in the humidity-controlled glove box (Xu et al., 2018). Xu et al., 2018 do not report analyses confirming the salt composition or identify impurities.

\subsection{2. $\quad H e a t$ Capacity Measurements: Xu $2018 \mathrm{MgCl}_{2}-\mathrm{KCl}$ Data Set}

The melting point and specific heat capacity of the $\mathrm{MgCl}_{2}-\mathrm{KCl}$ samples were measured using a simultaneous differential scanning calorimetry and thermogravimetric analyses system (STA449 F3 NETZSCH). The calibration of the instrument is discussed in Dehghani et al., 2016. Ten separate melting point and heat capacity measurements with fresh $32-68$ mole $\% \mathrm{MgCl}_{2}-\mathrm{KCl}$ samples were performed. The results show consistency between samples with a release of water vapor from $100{ }^{\circ} \mathrm{C}-200{ }^{\circ} \mathrm{C}$ and the onset of melting at around $424^{\circ} \mathrm{C}$ (Xu et al., 2018). The average measured melting temperature was $424.4^{\circ} \mathrm{C}$ with a relative error of 1.3\% (Xu et al., 2018). The heat capacity results are summarized in Figure 2. The data points in Figure 2 represent the mean values of measurements with ten fresh salt samples. The red error bars show the standard deviation and the green error bars show the overall measurement uncertainty.

The standard deviation and measurement uncertainty of the mean heat capacity value from the ten independent measurements were quantified using the following equations:

$S_{\bar{X}}=\frac{S_{X}}{\sqrt{N}}=\sqrt{\frac{\sum_{i=1}^{N}\left(X_{i}-\bar{X}\right)^{2}}{N(N-1)}}$

$u_{\bar{X}}=t_{N}-1.95 \% S_{\bar{X}}$ 
where $S$ is the standard deviation, $X$ is an individual heat capacity at a specified temperature, $\bar{X}$ is the mean of all heat capacity measurements, $N$ is the number of tests (10), $u$ indicates uncertainty and $T_{N^{-}} 1.95 \%$ is the Student's t distribution number, which is 2.262 for $N=10$ at the $95 \%$ confidence interval (Xu et al., 2018).

The uncertainty of the curve fitting for the heat capacity data was calculated using the following standard relationship:

$u_{c}=t_{v, 95 \%} \frac{S_{c}}{\sqrt{N}}=t_{v, 95 \%} \sqrt{\frac{\sum_{i=1}^{N}\left(C p_{i}-C p_{c i}\right)^{2}}{v N}}$

where $S_{c}$ is the standard deviation for the polynomial correlation, $C p_{i}$ is the measured heat capacity, $C p_{c i}$ is the heat capacity calculated from the polynomial, $v=N-(m+1)$ and $m$ is the order of the polynomial fit.

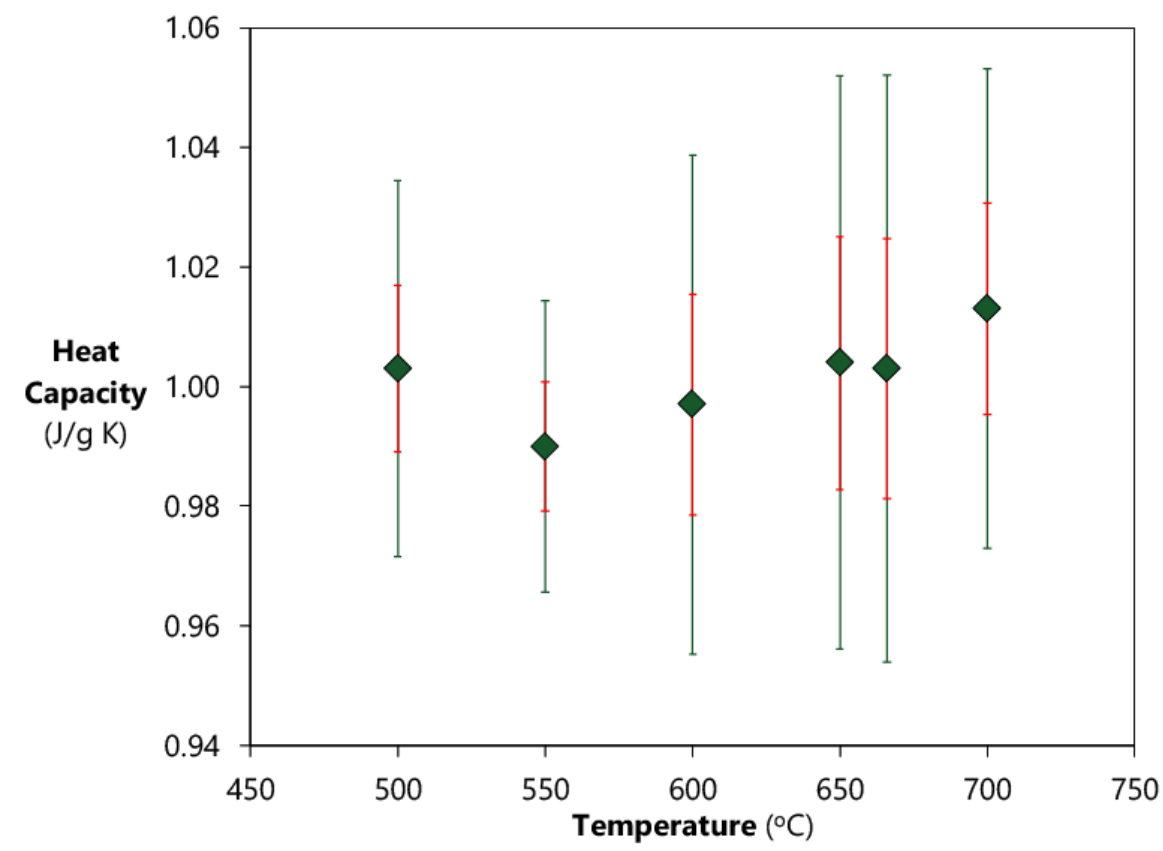

Figure 2. Heat capacity measurements (mean values) from the $\mathrm{Xu}$ et al., 2018 data set. The red error bars indicate the standard deviation for multiple measurements at the same temperate and the green error bars indicate the overall uncertainty as defined by Equations 1 and 2.

\subsubsection{Viscosity Measurements: Xu $2018 \mathrm{MgCl}_{2}-\mathrm{KCl}$ Data Set}

The viscosity of the $\mathrm{MgCl}_{2}-\mathrm{KCl}$ samples was measured using a custom designed high temperature rotational (Brookfield) viscometer. The accuracy of the viscometry measurements was quantified by running known salt mixtures as a function of temperature (documented in Wang et al., 2014). The viscometer consistently showed a less than $1 \%$ difference between measured data and known salts during calibration runs (Wang et al., 2014). 
Four tests, each with fresh salt, were performed and the repeatability of the measurements was deemed acceptable (Xu et al., 2018). The mean measured viscosity values as well as standard deviations and measurement uncertainties are shown in Figure 3. The standard deviations and uncertainties were calculated using Equations 1 and 2. The results show the salt viscosity decreases with increasing temperature with values less than 4 centipoise at temperatures higher than $600{ }^{\circ} \mathrm{C}$ (Xu et al., 2018).

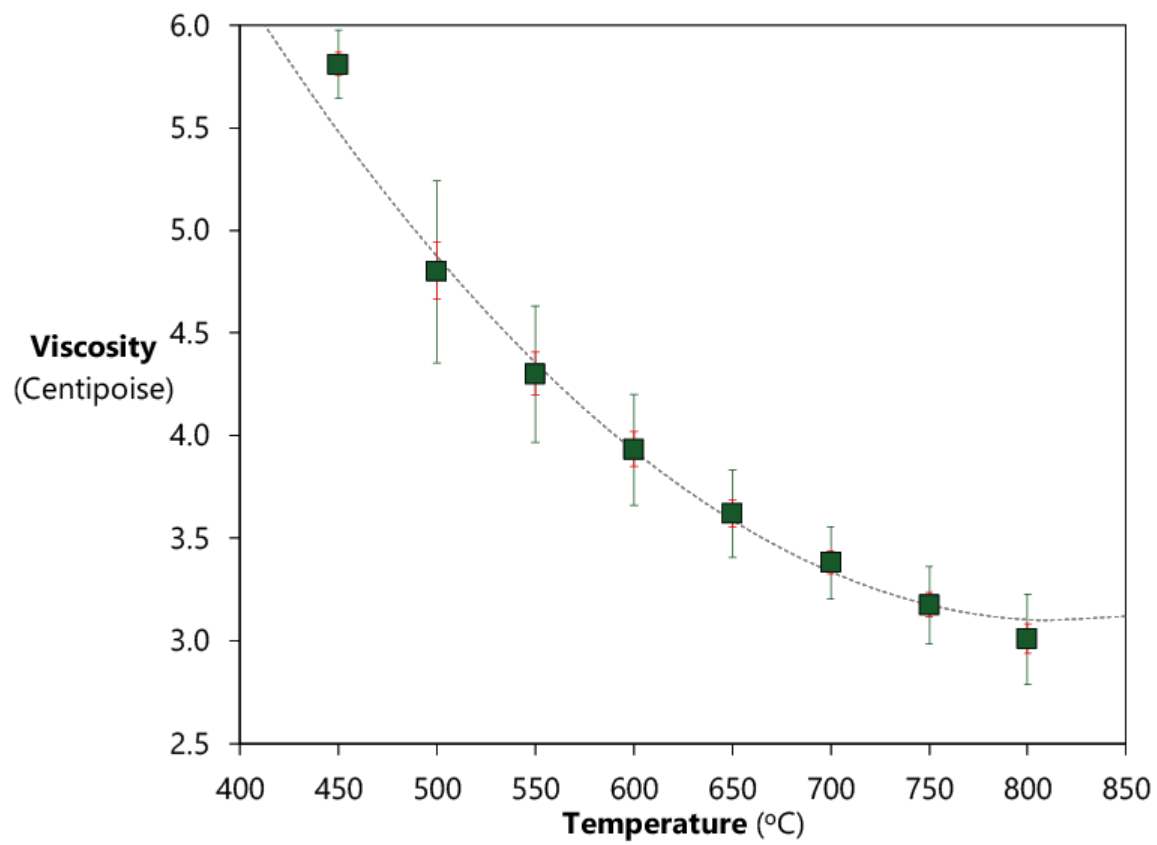

Figure 3. Viscosity measurements (mean values) from the Xu et al., 2018 data set. The red error bars indicate the standard deviation for multiple measurements at the same temperate and the green error bars indicate the overall uncertainty as defined by Equations 1 and 2.

\subsubsection{Density Measurements: $\mathrm{Xu} 2018 \mathrm{MgCl}_{2}-\mathrm{KCl}$ Data Set}

The density of the $\mathrm{MgCl}_{2}-\mathrm{KCl}$ samples were measured using a custom designed Archimedean density meter (Xu et al., 2018). The calibration of the meter is discussed in detail in Li et al., 2016. The density of pure $\mathrm{NaCl}$ and $\mathrm{KCl}$ were measured at $1200{ }^{\circ} \mathrm{C}$ and the density meter showed less than $1 \%$ difference between the measured values and established literature vales for these salts. Five density measurements, each with fresh salt, were made and the results showed good repeatability (Xu et al., 2018). The mean density measurements, standard deviations and measurement uncertainties for the $\mathrm{MgCl}_{2}-\mathrm{KCl}$ tests are shown in Figure 4. 


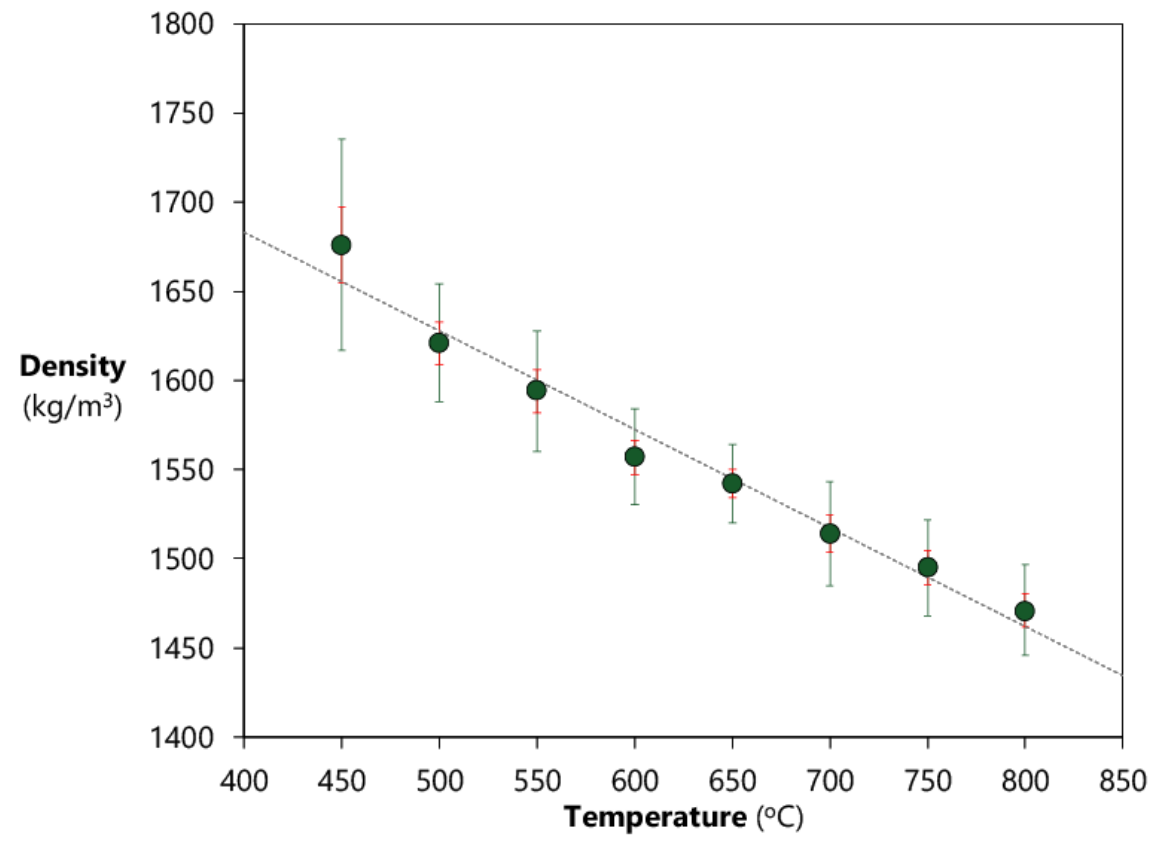

Figure 4. Density measurements (mean values) from the Xu et al., 2018 data set. The red error bars indicate the standard deviation for multiple measurements at the same temperate and the green error bars indicate the overall uncertainty as defined by Equations 1 and 2.

\subsubsection{Thermal Conductivity Measurements: Xu $2018 \mathrm{MgCl}_{2}-\mathrm{KCl}$ Data Set}

Thermal conductivity for the $\mathrm{MgCl}_{2}-\mathrm{KCl}$ salt was measured using a Laser Flash Analyzer (NETZSCH 457 MicroFlash). The calibration of this instrument, as discussed in Li et al., 2016, involved measuring the thermal conductivity of a known salt mixture (46-54 mole \% $\mathrm{NaNO}_{3}-\mathrm{KNO}_{3}$ ). The difference between the measured values and established values for the $\mathrm{NaNO}_{3}-\mathrm{KNO}_{3}$ was found to be around $\pm 15 \%$ (Li et al., 2016). The method actually measures thermal diffusivity, which can be used to calculate thermal conductivity if the specific heat capacity and density of the samples are known for the temperature of interest. The relationship is as follows:

$k=\alpha \rho C_{P}$

where $k$ is the thermal conductivity, $\alpha$ is thermal diffusivity, $\rho$ is density and $C_{p}$ is heat capacity.

Ten thermal diffusivity measurements, each with fresh salt, were made at each temperature and all show satisfactory repeatability ( $\mathrm{Xu}$ et al., 2018). The mean values, standard deviations and measurement uncertainties are shown in Figure 5. The thermal diffusivity results show only a minor variation of 0.27 to $0.29 \mathrm{~mm}^{2} / \mathrm{W}$ over the $450-800{ }^{\circ} \mathrm{C}$ temperature range of interest.

To determine the measurement uncertainty of the derived thermal conductivity values, Equation 4 must be used to define an error propagation expression as shown below: 
$\frac{u_{\bar{k}}}{\bar{k}}=\frac{u_{\bar{\alpha}}}{\bar{\alpha}}+\frac{u_{\bar{\rho}}}{\bar{\rho}}+\frac{u_{\overline{C_{p}}}}{\overline{C_{p}}}$

where $u$ is uncertainty, $\bar{\alpha}$ is the mean thermal diffusivity from multiple analyses, $\bar{\rho}$ is the mean density from multiple analyses and $\overline{C_{p}}$ is the mean heat capacity from multiple analyses. The thermal conductivity results for the $\mathrm{MgCl}_{2}-\mathrm{KCl}$ samples as well as the overall measurement uncertainties derived from Equation 5 are shown in Figure 5.

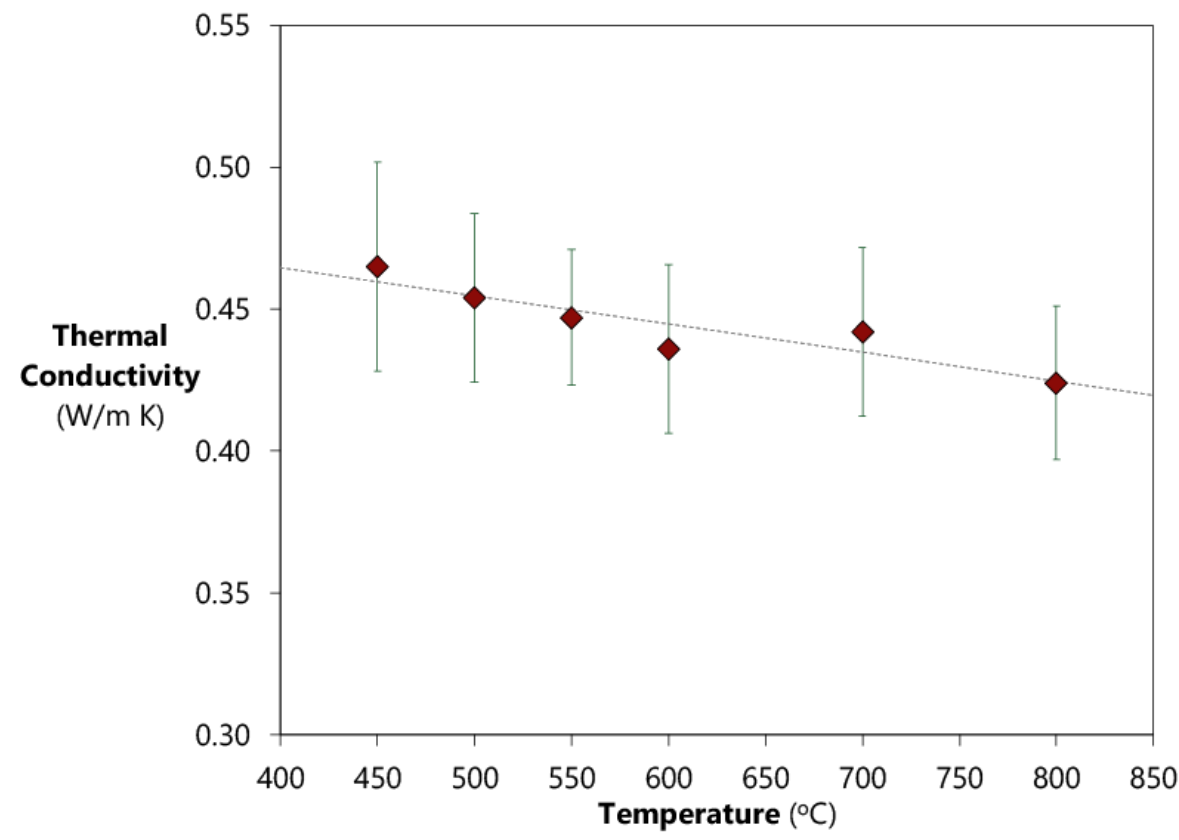

Figure 5. Thermal conductivity measurements (mean values) from the $\mathrm{Xu}$ et al., 2018 data set. The green error bars indicate the overall uncertainty, the standard deviation error bars are smaller than the data points.

\subsection{Cantor 1973, ORNL-TM-4308 Data Set}

Cantor measured the density and viscosity of several actinide and zirconium-bearing beryllium fluoride salts for use in molten salt reactors. Multiple measurements for each sample were performed and the overall uncertainty was estimated. Examples from this data set and empirical correlations that are used as input to the MSR thermophysical database are discussed below.

\subsection{1. $\quad$ Sample Preparation and Purity: Cantor 1973, ORNL-TM-4308 Data Set}

The salt mixtures $\mathrm{LiF}-\mathrm{BeF}_{2}$ (66-34 mole \%) and $\mathrm{LiF}_{-} \mathrm{BeF}_{2}-\mathrm{ZrF}_{4}(64.7-30.1-5.2$ mole \%) were made by mixing dry powders and melting within a helium atmosphere glovebox. The melts were purified by sparging with $\mathrm{H}_{2}-\mathrm{HF}$ gas (for information on sparging method see Shaffer, 1971). Samples of LiF-BeF $-\mathrm{ZrF}_{4}-\mathrm{UF}_{4}$ (64.79-29.96-4.99-0.26 mole \%) and samples $\mathrm{LiF}_{-} \mathrm{BeF}_{2}-\mathrm{ThF}_{4}$ with varying compositions were made by 
adding purified solid $\mathrm{LiF}, \mathrm{UF}_{4}$ and $\mathrm{LiF}_{-} \mathrm{ThF}_{4}$ (Cantor, 1973). Analytical-grade $\mathrm{KNO}_{3}$ was used as a reference material for checking the accuracy of the density measurements.

\subsubsection{Density Measurements: Cantor 1973, ORNL-TM-4308 Data Set}

Density measurements were made using a nickel dilatometer in which a metal probe detects changes in the salt liquid level within the neck of a volume-calibrated metal vessel (the method is described in Cantor, 1969). Measurements were taken at a pressure of $5 \mathrm{~atm}$ (argon gas) to minimize the volume of gas bubbles present within the liquid salt samples. Samples were weighed before and after density measurements to determine mass losses during tests. The weight change for all tests was less than $0.05 \%$. The dilatometer was recalibrated with distilled water at room temperature prior to each test. Analytical grade $\mathrm{KNO}_{3}$ of known density was used to check the measurements at higher temperatures (Cantor, 1973). The salt sample melt temperatures were controlled to within $\pm 0.2{ }^{\circ} \mathrm{C}$ (Cantor, 1973).

Results from the density measurements of Cantor, 1973 are shown in Figure 6 and the equations for the fits (dotted lines on Figure 6) are shown in Table 7. The standard errors in the density measurements are approximately $1.0 \mathrm{~kg} / \mathrm{m}^{3}$, that is around $0.05 \%$. Other sources of error include thermal creep of the dilatometer vessel, the volume of bubbles in the salt melt, and volatility indicated by small amounts of condensed salt within the vessel neck. A total error of $\pm 0.3 \%$ (accounting for the error sources just mentioned) was determined by comparing the measured $\mathrm{KNO}_{3}$ density at the test temperatures with the established literature values (Cantor, 1973).

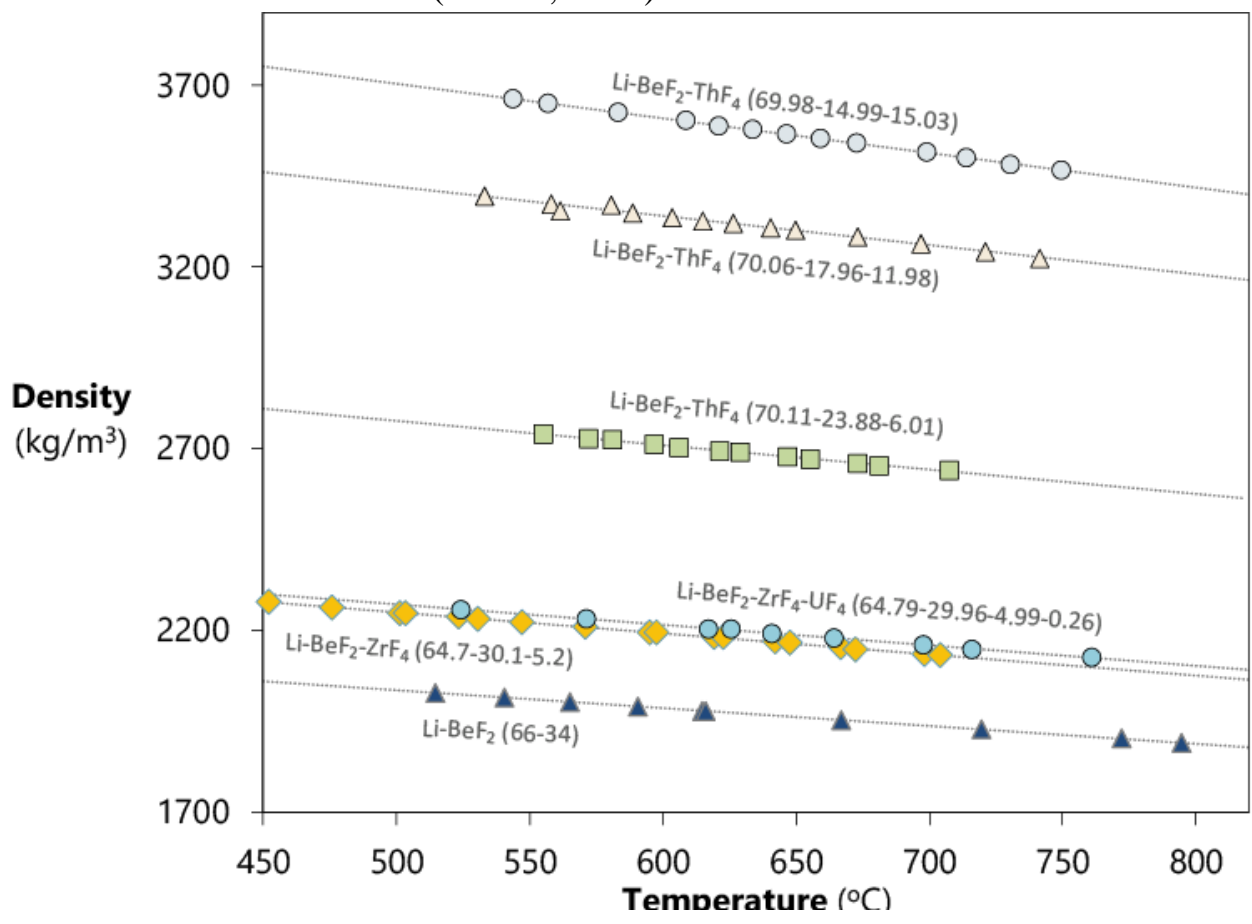

Figure 6. Density data from the Cantor 1973 dataset. The total errors for these measurements are approximately the same size as the data point symbols. The equations for the trendlines are shown in Table 7. 
Table 7. Density correlations derived from the data of Cantor, 1973 (Figure 6).

\begin{tabular}{|l|c|c|}
\hline Salt & \multicolumn{2}{|c|}{$\begin{array}{c}\text { Density Equation }\left(\mathbf{k g} / \mathbf{m}^{3}\right) \\
\end{array}$} \\
\hline & $\mathrm{A}$ & $\mathrm{B}$ \\
\hline $\mathrm{Li}-\mathrm{BeF}_{2}-\mathrm{ThF}_{4}(70.11-23.88-6.01)$ & 3.1118 & $6.707 \times 10^{-4}$ \\
\hline $\mathrm{Li}-\mathrm{BeF}_{2}-\mathrm{ThF}_{4}(70.06-17.96-11.98)$ & 3.8236 & $8.064 \times 10^{-4}$ \\
\hline $\mathrm{Li}-\mathrm{BeF}_{2}-\mathrm{ThF}_{4}(69.98-14.99-15.03)$ & 4.1811 & $9.526 \times 10^{-4}$ \\
\hline $\mathrm{Li}-\mathrm{BeF}_{2}(66-34)$ & 2.279 & $4.884 \times 10^{-4}$ \\
\hline $\mathrm{Li}-\mathrm{BeF}_{2}-\mathrm{ZrF}_{4}(64.7-30.1-5.2)$ & 2.5387 & $5.769 \times 10^{-4}$ \\
\hline $\mathrm{Li}-\mathrm{BeF}_{2}-\mathrm{ZrF}_{4}-\mathrm{UF}_{4}(64.79-29.96-4.99-0.26)$ & 2.5533 & $5.620 \times 10^{-4}$ \\
\hline
\end{tabular}

\subsubsection{Viscosity Measurements: Cantor 1973, ORNL-TM-4308 Data Set}

An oscillating-cup viscometer was used to measure the viscosity of $\mathrm{Li}_{-} \mathrm{BeF}_{2}-\mathrm{ThF}_{4}$ (67.27-15.7-11.6) and $\mathrm{Li}-\mathrm{BeF}_{2}-\mathrm{ThF}_{4}(70.11-23.88-6.01)$ at temperatures between $530-670{ }^{\circ} \mathrm{C}$. In this method, the salt is contained within a heated cylindrical vessel that undergoes torsional oscillations. The rate at which the amplitude of these oscillations is damped depends on the viscous drag of the salt on the walls of the vessel. The viscosity can be calculated from the damping rate of the system, the period of the oscillation, the dimension of the vessel and the mass and density of the salt melt at the test temperature by using standard equations. This method was developed for applications on non-aqueous high temperature liquids by Wittenberg and Ofte, 1970. The specific instrument and method used for the Cantor, 1973 measurements are described in Dewitt et al., 1974. Samples were prepared in a helium atmosphere glove box containing helium that had been passed through a charcoal trap maintained at liquid nitrogen temperature (Cantor, 1973).

Three measurements were made at each temperature for both salts. The mean and standard deviations for each test are shown in Figure 7. This plot also shows the power law correlation equations for each data set. The data show a decrease in viscosity of around 10 centipoise over the temperature range $520-670{ }^{\circ} \mathrm{C}$ for both salts. The salt with lower $\mathrm{BeF}_{2}$ and greater $\mathrm{ThF}_{4}$ has higher viscosity readings at every temperature (Cantor, 1973). 


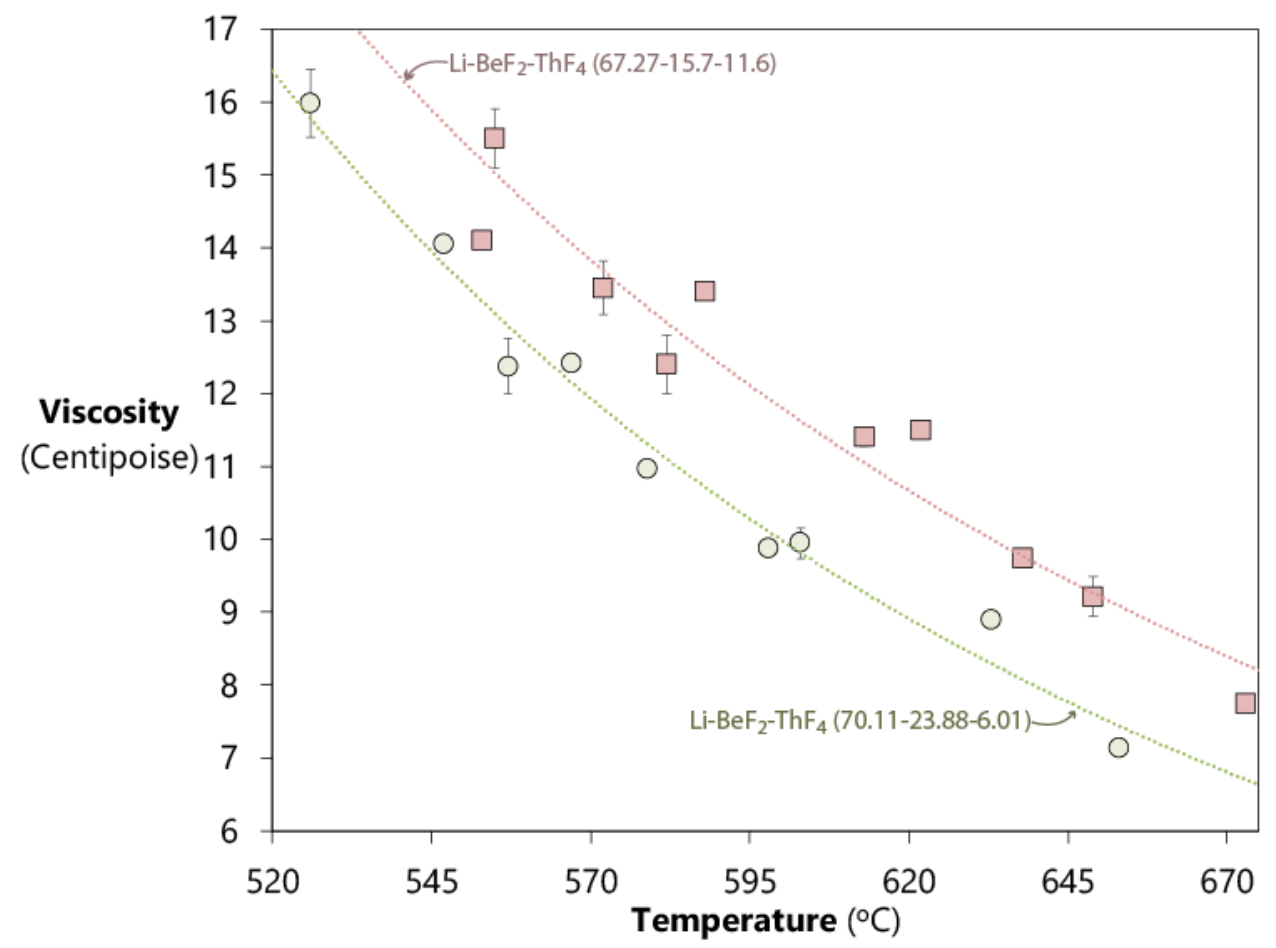

Figure 7. Viscosity measurements (mean values) from the Cantor 1973 data set. The error bars indicate the standard deviation of three measurements (some error bars are smaller than the datapoint symbols).

\subsection{Cohen and Jones 1957, ORNL-2278 Data Set}

In the mid-1950s Cohen and Jones measured the viscosity, density, heat capacity and thermal conductivity of 36 binary, ternary and quaternary fluoride salts (mostly actinide bearing) for the ORNL Aircraft Nuclear Propulsion Program. Of these data sets, the viscosity data have been retrieved and entered into the MSR Thermophysical Property database. The other property measurements are the subject of an ongoing critical literature review.

After reviewing several available methods for viscosity measurement, Cohen and Jones, 1957 decided to pursue both the rotational Brookfield viscometry method described by Redmond, 1952 and the capillary efflux viscometry method described by Tobias, 1951. Sample mixing and viscosity measurements were performed in an argon atmosphere dry box that housed a furnace set-up that accommodated both the capillary and rotational viscometers (Cohen and Jones, 1957). Measurements were made using both the rotational viscometer and capillary viscometer on the same sample of salt with tests being performed in series during a single heat-up cycle of the furnace. The total volume of salt used for the two instruments was around $100 \mathrm{~cm}^{3}$ (Cohen and Jones, 1957).

The efflux capillary viscometer consisted of a salt melt reservoir $\left(6 \mathrm{~cm}^{3}\right)$ connected to a capillary tube. The time of efflux is obtained as the salt melt drains through the capillary and compared with the efflux times of fluids of known viscosity. The reported precision for several efflux time measurements at a single 
temperature were $\pm 2-3 \%$ (Cohen and Jones, 1957). This method provides the kinematic viscosity of the fluid in centistokes which is converted to dynamic (absolute) viscosity using the following expression:

$\mu=v \cdot \rho$

where $\mu$ is the dynamic or absolute viscosity (centipoise $=10^{-3} \mathrm{~N} \mathrm{~s} / \mathrm{m}^{2}$ ), $v$ is the kinematic viscosity (in centiStokes $\left.=10^{-6} \mathrm{~m}^{2} / \mathrm{s}\right)$, and $\rho$ is density $\left(\mathrm{kg} / \mathrm{m}^{3}\right)$.

The Brookfield viscometer consisted of a rotating spindle that is immersed in the molten salt. Resistance to the torque applied to the spindle due to the salt viscosity is measured and converted to an absolute viscosity measurement in centipoise. The accuracy of the Brookfield viscometry measurements are significantly affected by the centering of the spindle during the test, the leveling of the spindle motor and ensuring a uniformity of temperature throughout and just above the salt sample (Cohen and Jones, 1957).

The measurements with the Brookfield viscometer were shown to be repeatable at a single temperature with variations generally within \pm 0.1 centipoises (Cohen and Jones, 1957). Calibration of both the capillary and Brookfield viscometers were made first with glycerol-water solutions having known viscosities and then with the ternary salt $\mathrm{NaNO}_{2}-\mathrm{NaNO}_{3}-\mathrm{KNO}_{3}$ (40-7-53 weight \%), which has both density and viscosity values within the range of fluoride salts at moderate temperatures ( 14.5 centipoises at $160{ }^{\circ} \mathrm{C}$ to 2 centipoises at $\left.400{ }^{\circ} \mathrm{C}\right)($ Cohen and Jones, 1957). Any time a new capillary reservoir or spindle was used, a full calibration curve specific to that device was generated. Furthermore, two or three new calibration points were measured after every set measurements of unknowns were made to ensure that the instrument had not drifted during the experiment (Cohen and Jones, 1957). Comparison of the two methods for both the measurements of calibration standards and the unknown fluoride salts consistently agree to within $\pm 10 \%$ (Cohen and Jones, 1957).

The results of Cohen and Jones, 1957 show that the all of the viscosity data measured can be fitted using equations of the form:

$\mu=A e^{B / T(K)}$

where $\mu$ is absolute viscosity, $T$ is temperature, and $A$ and $B$ are empirical parameters. The measured data and empirical parameters from the Cohen and Jones tests are shown in Table 8. The data represent mean values for several measurements at each temperature using both methods. The individual measurements are not provided in the Cohen and Jones, 1957 report. The overall accuracy of the measurements is estimated to be $\pm 10 \%$ based on comparisons of data for the completely independent viscometry methods that were used.

A comparison between the viscosity data from the Cantor, 1973 and the Cohen and Jones, 1953 datasets can be made for the lithium, beryllium actinide fluoride salt samples shown on Figure 8. Although the compositions of these salts are somewhat different, the results show that the Cohen and Jones, 1953 data are consistent with the trendline for the Cantor, 1973 data (Figure 8). This observation lends confidence in the measured viscosities, as the measurements were made using different techniques in different laboratories. 


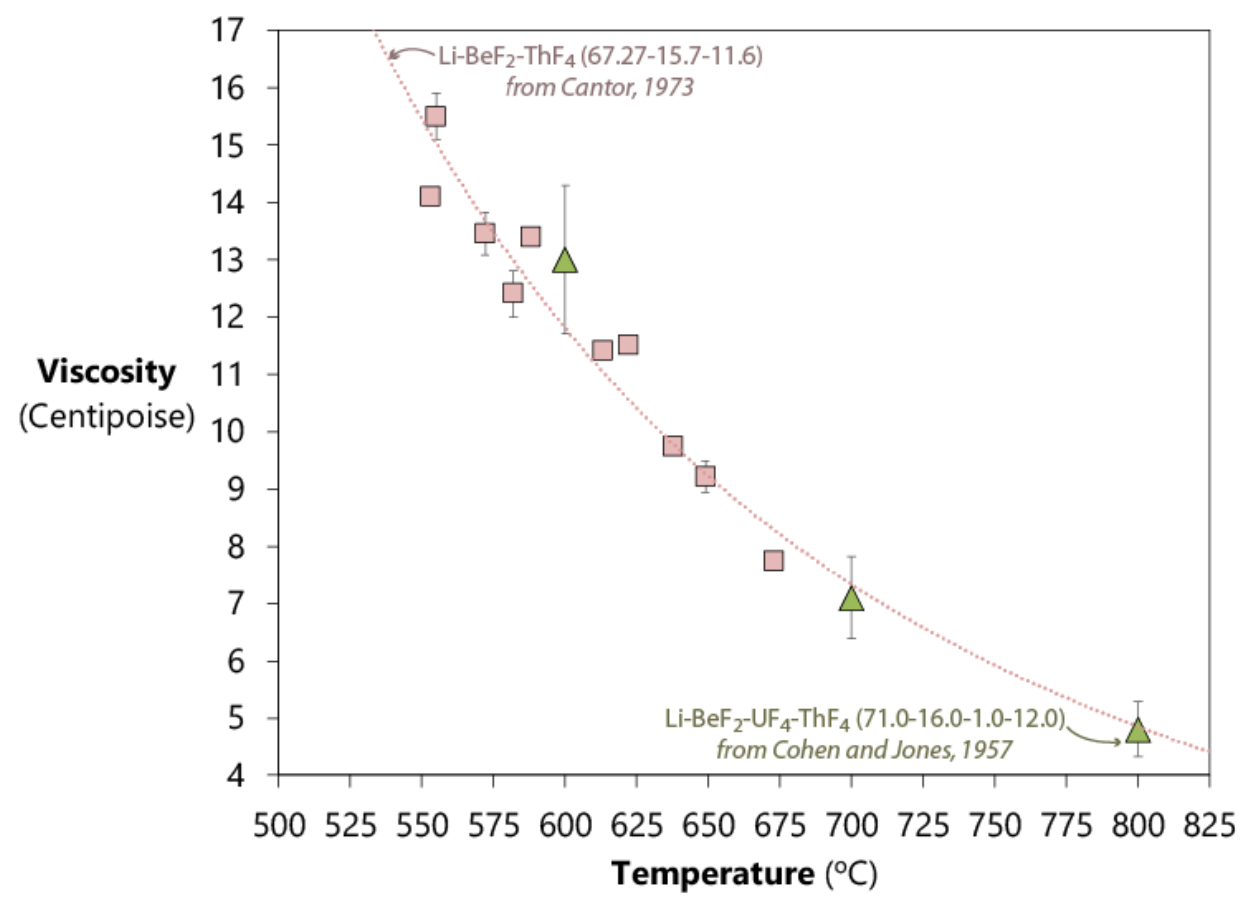

Figure 8. Viscosity data from the Cantor, 1973 data set and from the Cohen and Jones 1957 dataset. 
Table 8. Compositions, mean viscosity measurement values and correlation parameters for the Cohen and Jones, 1957 data set.

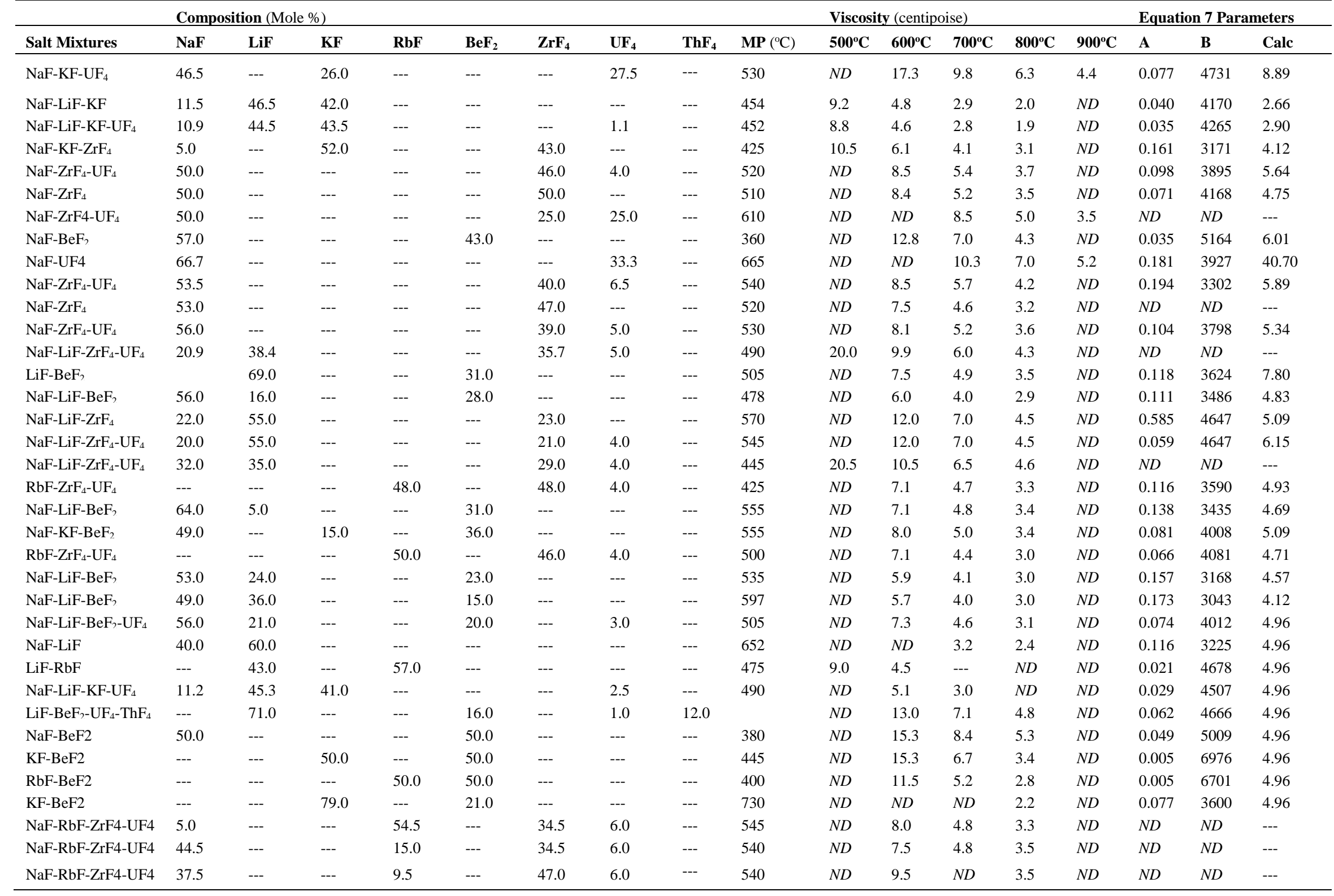

$N D$ : not determined. 


\section{Conclusions and Future Work}

The review of three molten salt thermophysical properties studies indicates that data for some key properties for MSR-relevant salts exist in the open literature. However, the existing literature data sets are sparse and incomplete for the actinide-bearing salts relevant to modern MSR applications. It is concluded that there remains a need for an experimental program focused on collecting data needed to address the design and licensing needs of current MSR concept developers. This experimental program would fill gaps in the existing salt properties database (see Tables 2 and 3 above) and provide data to derive and validate analytical expressions used for interpolation and extrapolation of property values for compositionally complex salts (e.g., fission product- and actinide-rich salts). The following list identifies the main properties that need to be quantified to support modeling MSR systems and represent the core salt properties addressed in a functional thermophysical properties database. Also listed are the measurement methods recommended in recent literature reviews (Serrano-López et al., 2013, Capelli, 2015; Fortner et al., 2018).

- Salt composition, phase equilibria

- Differential Scanning Calorimetry (DSC),

- Differential Thermal Analysis (DTA)

- Mass Spectrometry (MS)

- Heats of fusion

- DSC, other calorimetric methods

- Heat capacities

- DSC, other calorimetric methods

- Thermal conductivity

- Laser flash method (thermal diffusivity)

- Density

- Archimedes method

- Vapor pressure

○ Knudsen effusion

- Transpiration

- Volume Expansion

- Archimedes method

- Viscosity

○ Rotating spindle viscometer,

- Oscillating cup viscometer

There is also a need to continue to strengthen the technical link between the MSR modeling and simulation work and the experimental thermophysical properties measurement work. For example, MSR models could provide sensitivity runs to rank the relative importance of particular salt systems and thermophysical properties so that the experimental work can be prioritized and more directly linked to the modeling and simulation work needs. 


\section{References}

Auger, T., Barreau, G., Chevalier, J., Doligez, X., Delpech, S., Flocard, H., Haas, B., Heuer, D., MerleLucotte, E., The CNRS research program on the thorium cycle and the Molten Salt Reactors" (PACEN CNRS, Summary of Activities) June 2008

Beneš, O., Konings, R. J. M., Thermodynamic properties and phase diagrams of fluoride salts for nuclear applications, J. Fluor. Chem. 130 (2009)

Cantor, S., Metal Dilatometer for Determining Density and Expansivity of Volatile Liquids at Elevated Temperature, Rev. Sci. Instr. 40, 967 (1969).

Capelli, E., Thermodynamic Characterization of Salt Components for Molten Salt Reactor Fuel, Doctoral Dissertation, Delft University of Technology, the Netherlands, (2013).

Dehghani, G., Xu, X. K., and Li, P. W., 2016, Measurement of the Basic Properties of Ternary Eutectic Chloride Salts Used as High Temperature Heat Transfer Fluids and Thermal Storage Media, ASME Paper No. ES2016-59190.

Dewitt, R., Layton J. Wittenberg L., J., Cantor, S., Viscosity of molten NaCI, $\mathrm{NaBF}_{4}$ and $\mathrm{KBF}_{4}$, Physics and Chemistry of Liquids, 4:2-3, 113-123, (1974).

Forsberg, C. W., Peterson, P., Kochendarfer R., Design options for the Advanced High-Temperature Reactor (Paper 8026 ICAPP, Anaheim, CA USA, 8th-12th June, 2008)

Furukawa, K., Kato Y., Kamei, T., Developmental strategy of THORIMS-NES consisted of Th-MSR "FUJI" and AMSB IAEA- TU2007, Beijing, (2007)

Grimes, W. R., Chemical research and development for the Molten-Salt Breeder Reactor ORNL/TM-1853 (1967)

Holcomb, D. E., Flanagan, G. F., Patton, B. W. Gehin, J. C., Howard, R. L., Harrison, T. J., Fast Spectrum Molten Salt Reactor Options, ORNL/TM-2011/105, July 2011

Ignatiev V., Merzlyakov A., Transport Properties of Molten Salt Reactor Fuel Mixtures, paper presented at Actinide and Fission Product Partitioning Transmutation - 7th Information Exchange Meeting, Jeju, Korea, (October 14-16, 2002).

Ignatiev, V. Feynberg, O., Mjasnikov, A., Zakirov, R., Reactor physics and fuel cycle analysis of a Molten Salt Advanced Reactor Transmuter, International Congress on Advances in Nuclear Power Plants (ICAPP 2003)

Ingersoll, D.T., Status of preconceptual design of the Advanced High-Temperature Reactor ORNL/TM2004/104 (2004) 
Janz, G.J., Allen, C.B. Downey Jr., J.R., Tomkins, R.P.T., Physical properties data compilations relevant to energy storage. I. Molten salts: Eutectic Data, NSRDS-NBS-61 (Pt. I), U.S. National Bureau of Standards, 1978.

Janz, G.J., Allen, C.B., Bansal, N.P., Murphy, R.M., Tomkins, R.P.T., Physical properties data compilations relevant to energy storage II. Molten salts: data onsingle and multi-component salt systems, NSRDS-NBS61 (Pt. II), U.S. National Bureau of Standards, 1979.

Janz, G.J., Krebs, U., Siegenthaler, H.F., Tomkins, R.P.T., Journal of Physical and Chemical Reference Data 1 (1972) 581-746.

Janz, G.J., Gardner, G.L., Krebs, U., Tomkins, R.P.T., Journal of Physical and Chemical Reference Data 3 (1974) 1-115.

Janz, G.J., Tomkins, R.P.T., Allen, C.B., Downey Jr. J.R., Gardner, G.L, Krebs, U., Singer, S.K., Journal of Physical and Chemical Reference Data 4 (1975) 871-1178.

Janz, G.J., Tomkins, R.P.T., Allen, C.B., Downey Jr. J.R., Singer, S.K., Journal of Physical and Chemical Reference Data 6 (1977) 409-596.

Janz, G.J., Tomkins, R.P.T., Journal of Physical and Chemical Reference Data 12(1983) 591-815.

Janz, G.J., Tomkins, R.P.T., Journal of Physical and Chemical Reference Data 9(1980) 831-1021.

Janz, G.J., Tomkins, R.P.T., Physical properties data compilations relevant to energy storage. IV. Molten salts: Data on Additional Single and Multi-Component Salt Systems, NSRDS-NBS-61 (Pt. IV), U.S. National Bureau of Standards, 1981.

Janz, G.J., Dijkhuis, C.G.M., Lakshminarayanan, G.R., Tomkins, R.P.T., Wong, J., Molten Salts: Volume 2. Section 1. Electrochemistry of Molten Salts, GibbsFree Energies and Excess Free Energies from Equilibrium-Type Cells, Section2. Surface Tension Data, NSRDS-NBS-28, U.S. National Bureau of Standards, 1969.

Janz, G.J., Journal of Physical and Chemical Reference Data 17 (1988) 1-309.

Janz,G.J., Dampier, F.W., Lakshminarayanan, G.R., Lorenz, P.K., Tomkins, R.P.T., Molten salts: Volume 1, Electrical Conductance, Density, and Viscosity Data,NSRDS-NBS-15, U.S. National Bureau of Standards, 1968.

Khokhlov, V., Ignativ, V., Afronichkin, V., Evaluating physical properties of molten salt reactor fluoride mixtures, J. Fluor. Chem. 130 (2009) 30

Latge, C., Alternative coolants for intermediate loops of innovative SFR (Presentation from CEA, Cadarache, for ALISIA Info Meeting, Paris, France, 4th March, 2008) 
Li, P. W., Molina, E., Wang, K., Xu, X., Dehghani, G., Kohl, A., Hao, Q., Kassaee, M. H., Jeter, S. M., and Teja, A. S., 2016, "Thermal and Transport Properties of $\mathrm{NaCl}-\mathrm{KCl}-\mathrm{ZnCl}_{2}$ Eutectic Salts for New Generation High-Temperature Heat-Transfer Fluids,” ASME J. Sol. Energy Eng., 138(5), p. 054501.

Shaffer, J. H., Preparation and Handling of Salt Mixtures for the Molten Salt Reactor Experiment, ORNL4616 (January 1971).

Sohal, M., S., Ebner, M., A., Sabharwall, P., Sharpe, P., Engineering Database of Liquid Salt Thermophysical and Thermochemical Properties, INL/EXT-10-18297, Rev. 1, June 2013

Mourogov, A., Bokov, P.M., Energ. Convers. Manage. 47 (2006) 2761.

Redmond, R. F., ANP Quarterly Progress Report for Period Ending June 10, 1952, ORNL-1294 p. 124.

Renault, C., Delpech, M., 2005, "Review of Molten Salt Reactor Technology: MOST Final Report" European Commission, 5th Euratom Framework Programme (March, 2005)

Samuel, D., Molten Salt Coolants for High Temperature Reactors: A Literature Summary of Key R\&D Activities and Challenges, IAEA, Literature Summary Report (May, 2009)

Serrano-López, R., Fradera, J., Cuesta-López, S., Molten salts database for energy applications, Chemical Engineering and Processing 73 (2013) 87- 102

Tobias, M., ANP Quarterly Progress Report for Period Ending December 10, 1951, ORNL-1170 p. 124.

Wang, K., Molina, E., Dehghani, G., Xu, B., Li, P. W., Hao, Q., Lucas, P., Kassaee, M. H., Jeter, S. M., and Teja, A. S., 2014, "Experimental Investigation to the Properties of Eutectic Salts by NaCl-KCl-ZnCl2 for Application as High Temperature Heat Transfer Fluids," ASME Paper No. ES2014-6578.

Williams, D. F., Assessment of Candidate Molten Salt Coolants for the NGNP/NHI Heat-Transfer Loop, ORNL/TM-2006/69, Oak Ridge National Laboratory, Oak Ridge, TN (2006).

Williams, D. F., Toth, L. M., Clarno, K. T., "Assessment of candidate molten salt coolants for the Advanced High-Temperature Reactor (AHTR)" ORNL/TM-2006/12 (2006) (online)

Wittenberg L. J. and Ofte, D., Viscometry and Densitometry - A. Viscosity of Liquid Metals, Physicochemical Measurements in Metals Research, Part 2, R. A. Rapp (ed.), Interscience Publishers, New York, 1970, pp. 193-217.

Xu, X., Wang, X., Li, P., Li, Y., Hao, Q., Xiao, B., Elsentriecy, H., Gervasio, D., Experimental Test of Properties of $\mathrm{KCl}-\mathrm{MgCl}_{2}$ Eutectic Molten Salt for Heat Transfer and Thermal Storage Fluid in Concentrated Solar Power Systems Journal of Solar Energy Engineering, OCTOBER 2018, Vol. 140 


\section{Argonne}

Chemical and Fuel Cycle Technologies Division

Argonne National Laboratory

9700 South Cass Avenue, Bldg. 205

Argonne, IL 60439

www.anl.gov 